\title{
Elevage en forêt dans les Landes de Gascogne I. - Le système végétal
}

\author{
B. LEMOINE $*$, $* * *$, D. BONHOMME et D. CHINZI **, \\ B. COMPS, H. BERGERET ***, J. GELPE***;,

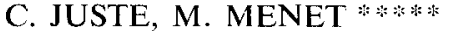 \\ * Responsable scientifique de la Recherche engagée avec l'aide de la D.G.R.S.T. \\ Comités G.R.N.R. et E.C.A.R. - Contrats : 76-77-78 \\ ** E.N.I.T.A., Chaire de Productions animales \\ 1, cours du Général-de-Gaulle, F 33170 Gradignan \\ *** Université de Bordeaux I, Laboratoire de Botanique \\ F 33405 Talence \\ ***** I.N.R.A., Laboratoire de Sylviculture et d'Ecologie de la Pinède landaise \\ Centre de Recherches de Bordeaux, Pierroton, F 33610 Cestas \\ *:**** I.N.R.A., Station d'Agronomie, Domaine de la Grande-Ferrade \\ Centre de Recherches de Bordeaux, F 33140 Pont-de-Maye
}

\section{Résumé}

L'objectif de ce travail est double : d'une part déterminer le potentiel fourrager des sous-bois en lande humide, d'autre part amorcer une étude du fonctionnement de cet ćcosystème à deux strates végétales — peuplement de pins et sous-bois.

Les principaux résultats sont les suivants :

a) les plantes fourragères cultivées sont peu compétitives vis-à-vis de la flore spontanée à base essentiellement de Molinia coerulea M., ou molinie ;

b) la molinie a une productivité modeste - entre 0,7 et 1,8 tonne de matière sèche/ha/an -, et n'est utilisable par l'animal que pendant 5 à 6 mois ; cette productivité varie en fonction de la densité clu peuplement forestier et est sensible à l'apport d'éléments minéraux - essentiellement $\mathrm{N}$ avec interaction de $\mathrm{P}$ et de $\mathrm{K}$; on envisage plutôt une utilisation extensive de ce sous-bois ;

c) les données de productions périodiques de l'herbe s'ajustent, de façon satisfaisante, selon une régression double aux variations des paramètres climatiques - ETP et déficit hydrique - modifiés par le peuplement d'arbres;

d) l'action de l'animal sur l'herbe est vue à travers une «séquence de pâturage »;

e) le peuplement d'arbres bénéficie, au moins globalement, de l'exploitation de la prairie artificielle par l'animal.

Paraîtront prochainement dans d'autres revues et sous le même titre les articles concernant les autres aspects de cette recherche :

II. - Valeur nutritive et alimentaire des fourrages. In Bulletin du C.R.Z.V. de Theix.

III. - Le potentiel disponible.

IV. - Les modèles d'élevage.

V. - Evolution récente des systèmes de production agricole dans les Landes de Gascogne. 
L'introduction de l'animal en forêt, si elle s'avère possible économiquement pour l'éleveur, amòne à se poser une première question : verra-t-elle simplement la juxtaposition d'un système d'élevage au système sylvicole existant, ou mieux la mise au point d'un système mixte optimisé ?

Dans le cas de la forêt landaise, l'intégration devra se faire en tenant compte de ses caractéristiques technicoéconomiques dont les traits suivants doivent être rappelés :

a) Sa productivité ligneuse est constamment en progression, d'abord grâce à la généralisation du drainage et du débroussaillage ces deux dernières décennies, puis aujourd'hui et demain grâce à la généralisation de la fertilisation (GelPE J. et al., 1974). On a affaire à un système très productif dans lequel le sylviculteur s'efforce de contrôler mécaniquement le sous-bois. Les travaux de l'Inventaire Forestier National et des organismes de recherches permettent de cerner la potentialité de la forêt de l'échelle du massif à celle de la parcelle. Son utilisation peut aussi évoluer sous la pression de contraintes économiques vers une diminution de la fréquence des éclaircies et de la durée de la révolution.

b) Sa fonction économique s'amplifie sur le plan national. Le forestier donne la priorité à la production de bois. Il s'agit donc plus, dans l'immédiat, d'accueillir l'éleveur que de composer avec lui en recherchant l'optimisation d'un système mixte. D'ailleurs ce ne serait que très rarement que ces deux partenaires se retrouveraient au sein de la même personne. Dès lors l'objectif de la recherche, donc les thèmes scientifiques abordés, pourrait se limiter au modèle de juxtaposition de deux productions. Mais nous avons été tentés par une prospective plus large pour deux raisons : d'une part l'éventualité qu'une innovation pourrait trouver à long terme une justifification socioéconomique, d'autre part qu'une recherche plus approfondie pourrait déboucher sur un modèle dont on aurait fait varier le maximum de paramètres, sans omettre en particulier le paramètre sylvicole, c'est-à-dire la densité du peuplement de pins ; ce modèle, ou tout au moins la méthodologie nécessaire à sa mise sur pied, pourrait servir ailleurs; il se trouvait que les Landes de Gascogne bénéficiaient sur place d'un potentiel de recherches pluridisciplinaires important.

Le sous-bois ne bénéficiait jusqu’à présent que de connaissances qualitatives à petite échelle (carte de la végétation : LEREDE C., 1947), qui permettent néanmoins de distinguer les zones de pelouses à molinie des différentes zones de landes, ce qui offre un intérêt certain pour la prospective élevage en forêt au niveau régional. A l'échelle plus grande du bassin versant, plus proche aussi de celle à laquelle l'éleveur doit opérer, un approfondissement de la typologie de la végétation en relation avec les facteurs du milieu s'est révélé nécessaire, ce qui a été rendu possible grâce à une action concertée D.G.R.S.T. antérieure (Comps et al., 1979). Cependant aucune donnée quantitative, c'est-à-dire ayant trait à la productivité du sous-bois, n'était disponible au démarrage de laction concertée «élevage en forêt».

Sur le plan de l'historique de la démarche, il est bon de rappeler que cette action pluridisciplinaire s'est appuyée sur une concertation déjà existante entre des organismes de recherche et de développement. C'est ainsi par exemple que l'élevage ovin expérimental de la C.A.R.A. à Cestas (Petit Jarry) fait encore l'objet d'observations ; on $\mathrm{y}$ a étudié les problèmes d'implantation de flore prairiale artificielle, de fertilisation et d'effets globaux sur l'arbre. Les résultats acquis dans ce cadre expérimental ont permis de mieux orienter les recherches à venir : c'est ainsi que l'étude du sousbois naturel se révélait absolument nécessaire. 


\section{Définition de la recherche}

Nous parlerons des niveaux d'approche et des thèmes étudiés.

\subsection{Niveaux d'approche}

Le niveau descriptif permet dans bien des cas d'aboutir à certaines conclusions d'ordre technicoéconomique. On peut prendre pour exemple en sylviculture celui des Tables de Production (N. Decourt et al., 1970). Parallèlement une connaissance touchant les potentialités du sous-bois sera de la plus haute utilité. Elle pourra se compléter par des observations sur les effets de la fertilisation ou de l'animal, par une mise en relation statistique entre les paramètres de production de la forêt (âge, densité, accroissements) et ceux de la production du sous-bois.

Le passage du niveau descriptif au niveau explicatif se justifie par plusieurs raisons. D'abord au niveau pratique on verrait mal par exemple comment définir les marges de sécurité ayant trait à un calendrier fourrager sans connaître la relation entre la productivité du sous-bois et les variations temporelles du climat et en conséquence sans passer par une modélisation. Ensuite sur le plan prospectif il coûte extrêmement cher de mettre en place des systèmes nouveaux alors que la compréhension des systèmes existants suivie de modélisation peut orienter commodément les études. N'oublions pas non plus que la prospective doit s’appliquer aussi aux équilibres écologiques et que de ce point de vue le niveau explicatif est absolument nécessaire.

C'est ainsi que nous avons opté pour des recherches offrant un compromis entre une compréhension suffisante des phénomènes observés et nos propres contraintes.

\subsection{Thèmes de recherches}

Pour le sujet, relativement complexe, qui nous occupe ici, l'analyse systémique (figure 1) revêt une importance primordiale. Bien sûr, il est impensable, faute de moyens suffisants, d'aborder tous les thèmes auxquels conduit cette analyse. Par exemple une étude bioclimatologique complète du fonctionnement du système à deux strates, l'arbre et le sous-bois, eût été extrêmement intéressante pour juger de l'économie énergétique de ce système, pris comme une culture associée, par rapport à des systèmes à une strate.

La compréhension des caractéristiques de croissance et de productivité du sousbois et plus généralement de la relation sous-bois - peuplement d'arbres doit passer par les concepts de concurrence et de coopération : une strate peut être favorable ou défavorable à l'autre. Mais ce type de phénomène doit être examiné en tenant compte des facteurs physiques pouvant jouer le rôle de facteurs limitants : l'eau, l'énergie, les éléments minéraux. On doit donc d'emblée faire l'hypothèse que le facteur physique pour lequel va s'exercer prioritairement la concurrence sera fonction du site écologique : concurrence pour l'eau en lande sèche, concurrence pour la lumière en lande humide. Dans le premier cas l'analyse des phénomènes se complique, puisque l'examen des systèmes racinaires (racines actives) de deux strates et la détermination de leur position relative semble s'imposer. 


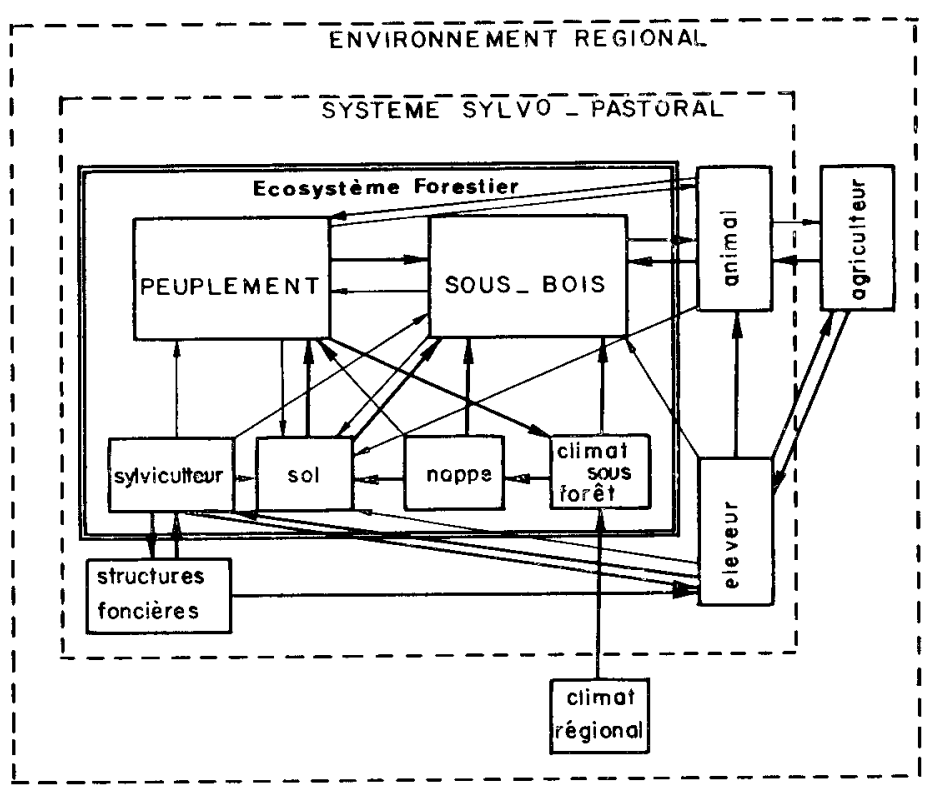

Fig. 1

Analyse du système.

Analysis of the system.

La variation du peuplement forestier, qui est le fait de sa dynamique de croissance et du type sylvicole, joue sans conteste sur les deux premiers facteurs physiques cités : l'eau et la lumière.

Face à toutes les questions posées, notre démarche a été la suivante :

1. Etudier plus particulièrement une plante et un écosystème :

Après avoir jugé des possibilités d'introduction de plantes fourragères en sousbois landais - ces possibilités sont faibles - et obtenu un premier modèle de réaction de l'herbe à l'ombrage (Fétuque), nous nous sommes penchés plus particulièrement sur l'écosystème de lande humide et sur la graminée sauvage Molinia coerulea $\mathbf{M}$. ou molinie qui en est très représentative.

2. Recueillir des données phénologiques et de productivité de la strate herbacée; les confronter ensuite aux paramètres du peuplement forestier (état et productivité); procéder à une première interprétation de cette relation biométrique en considérant la relation herbe-lumière (facteur présumé limitant en lande humide).

3. Procéder à un essai de généralisation d'un certain modèle de fonctionnement: ce modèle relie les accroissements en biomasses $\left(\frac{\Delta \mathrm{MS}}{\Delta \mathrm{t}}\right)$, soit à l'eau consommée 
ou ETR (JeanNin B., et al., 1979), soit à l'énergie et au déficit hydrique (DE Montard F., 1981); la modélisation en position de sous-bois implique de quantifier laction de la strate supérieure, ou peuplement forestier, sur les pluies (interceptions) et sur l'énergie, ainsi que la part de la réserve utile en eau $(\mathrm{Ru})$ qui revient à la strate inférieure.

4. Mener parallèlement des travaux d'écologie expérimentale sur la molinie afin de déterminer ses lois de réponse $\left(\right.$ en $\left.\frac{\Delta \mathrm{Ms}}{\Delta \mathrm{t}}\right)$ à leau (effets sécheresse et hydromorphie) et à la lumière; on simule en quelque sorte l'ombrage du peuplement forestier et différentes conditions hydriques, celles-ci encadrant largement les conditions que l'on peut rencontrer en position de sous-bois; cette recherche devrait permettre de vérifier les fondements des modèles de fonctionnement de la végétation en place.

5. Caractériser l'ambiance forestière de façon à pouvoir par la suite parfaire notre compréhension du fonctionnement du sous-bois par la voie du bilan d'eau et du bilan d'énergie. Il est nécessaire de recourir à la formule de Penman (BroCHet P., Gerbier N., 1970) en s’approchant au plus près des paramètres nécessaires au calcul de l'ETP : les mesures directes du rayonnement net sont nécessaires car on ne sait pas, si dans ces conditions particulières, on peut déduire avec suffisamment de précision ce paramètre du rayonnement global. Par ailleurs le rayonnement net nocturne (qui est négatif), pourrait caractériser les conditions du démarrage de la végétation du sous-bois. Quant à la fraction advective de l'ETP elle se mesure soit synthétiquement (évaporomètre de Piche sous abri), soit analytiquement ( 3 paramètres).

6. Caractériser l'action des éléments minéraux sur la production de molinie : deux démarches sont complémentaires et consistent, l'une à avoir recours à des expériences agronomiques afin de caractériser le ou les facteurs limitants, l'autre à caractériser la relation plante-éléments minéraux. La première démarche devrait déboucher sur une prospective culturale, la seconde sur l'approche de la relation herbe-animal (valeur fourragère, action des fécès sur l'herbe), ce qui permettrait d'amener un élément supplémentaire de généralisation du modèle cité plus haut (cf. § 3.).

7. Evaluer l'effet global de l'animal sur la croissance des arbres en utilisant les dispositifs semi-permanents; on a recours aux concepts de durée de pâturage et de «turnover» des éléments minéraux.

\section{RésuItats}

\subsection{Préambule : présentation des sites étudiés}

Les sols sont typiques de la lande humide de la région. Dans tous les sites ayant servi de base à l'expérimentation on est en présence de podzols humiques le plus souvent hydromorphes.

L'annexe I présente le Tableau floristique du site A de Pissos : on constate que 
la graminée Molinia coerulea M., la molinie, y est l'espèce dominante. Mais l'avoine de Thore, Pseudoarrhenaterum longifolium (Thore) R., lui est souvent associée dans des proportions parfois importantes, par exemple d'1/3 comme dans les sites $\mathrm{B}$ de l'Hermitage et $\mathbf{C}$ de Saint-Alban. On constate parfois aussi la présence de Pteris aquilina L., la fougère aigle, aux endroits moins humides, mésophiles, comme dans le site E du Petit Jarry. Les arbustes et arbrisseaux (bourdaine, ajoncs, bruyères...) sont le plus souvent rabattus, voire sérieusement endommagés par la pratique du débroussaillage.

\subsection{Possibilités d'introduction de plantes fourragères en sous-bois landais}

Pour accroître la production et la valeur nutritive de l'herbe du sous-bois, certains ont pensé détruire la végétation herbacée naturelle et lui substituer une véritable prairie artificielle.

Ceci était d’autant plus tentant dans la forêt de Gascogne que les techniques sylvicoles modernes impliquent le semis ou la plantation des pins en lignes ou en bandes. Elles conduisent également à travailler l'interligne de pins pour réduire ou éliminer la végétation adventice.

On peut dès lors envisager d'implanter, sur cette surface, des plantes fourragères à potentiel de production plus élevé que celui de la molinie ou de lavoine de Thore.

Dès 1965-1966, la C.A.L.G. (Compagnie d'Aménagement des Landes de Gascogne) avait réalisé une expérimentation sur une parcelle de pins en lande humide dans la commune de Luxey. Les résultats des deux premières années de cette culture d'herbe assez intensive (40 à 60 unités d'azote après chaque exploitation) étaient très encourageants mais ne furent pas poursuivis.

Ils justifièrent lintroduction de cette technique sur des surfaces importantes dans les domaines expérimentaux de la C.A.R.A. à Petit Jarry (site E) et Pissos (site A) dans une autre unité.

Parallèlement le C.T.G.R.E.F. mit en place en 1973 et 1974 à Petit Jarry et en 1976 à Pissos des collections fourragères après avoir réalisé en 1971 un essai à Cannex (Landes) avec le Syndicat Ovin.

En attendant un bilan technique et économique complet, les observations, mesures, prélèvements, analyses que nous avons effectués conduisent à proposer les conclusions provisoires suivantes :

a) la pérennité des espèces implantées est faible:

Cette constatation rejoint celle des différents auteurs étrangers qui la lient à la surface terrière des arbres et à la lumière reçue au sol. Sous pins le rôle des aiguilles, qui sécrètent des inhibiteurs de germination vis-à-vis d'un certain nombre de plantes, serait important (HaLles \& SumaN, 1952).

Dans les collections fourragères du C.T.G.R.E.F. à Pissos, après 4 années d'observations et de mesures, on constate un envahissement important par l'Agrostis Setacée, les ajoncs, la bruyère et récemment par la molinie dans presque toutes les 
parcelles. Seules la houlque et les agrostis semés ont une bonne persistance. Les autres espèces ont pratiquement disparu à l'exception des Fétuques rouges, de la Fétuque Ludion et du dactyle Lucifer dont il reste quelques éléments.

Cette disparition rapide de la flore implantée a été également constatée à Petit Jarry accentuée sans doute par un chargement excessif des parcelles les deux premières années. Elle a été enregistrée également à Pissos surtout dans les parcelles où le plan d'eau était mal maîtrisé.

b) La production annuelle permise en sous-bois, même la première et la deuxième année, est modeste (moins de $3 \mathrm{t}$ de matière sèche/ha).

c) La valeur nutritive des espèces implantées en sous-bois est comparable à celle de ces espèces cultivées en sol nu, mais leur teneur en matière minérale est plus faible.

d) L'implantation des graminées fourragères en sous-bois landais, étant donné les investissements exigés et les résultats modestes obtenus, ne doit être conseillée quavec une très grande prudence. Les praticiens n'envisagent d'ailleurs pas cette technique.

\subsection{Productivité et écologie de la pelouse de lande humide}

Pour caractériser la strate herbacée l'étude conjointe des deux espèces de graminées - molinie et avoine de Thore - est le plus souvent nécessaire. La mesure des biomasses doit aussi être assortie de paramètres phytoécologiques, par exemple la contribution spécifique (DE VRIES, in BERGERET H., 1980) soit :

- la contribution spécifique au recouvrement de la surface totale ou C.S.s., qui tient compte des points «occupés» par la terre nue et la litière;

- la contribution spécifique au végétal vert total ou C.s. V.v.

Nous ne pouvons prétendre ici cerner complètement l'aspect statistique ayant trait à l'évaluation de la productivité. Une étude de la variabilité spatio-temporelle demanderait la mise en place de moyens plus importants et permanents. C'est pour cela que l'on a privilégié l'aspect «fonctionnement» sur quelques sites.

\subsection{Croissance et phénologie de la molinie}

La molinie a pu être observée dans le site A de Pissos (élevage expérimental bovin de la C.A.R.A.) dans différentes situations (placeaux ayant été antérieurement fertilisés ou non, pâturés ou non). Les traits dominants des années 1977 et 1978 sont les suivants (BERGERET H., 1980) :

- la feuille commence sa croissance début mars, atteint la moitié de sa hauteur finale en juin et culmine à $50 \mathrm{~cm}$ fin juillet ; bre ;

- le début de la montaison se situe en juin et l'épiaison est terminée en septem-

- la biomasse sur pied (matière sèche des parties épigées vivantes) culmine en juillet autour de $1800 \mathrm{~kg} /$ ha ; la figure 2 présente des courbes de croissance pondérale cumulée pour l'année 1977 ; en 1978 on observe des résultats sensiblement différents 
par rapport à 1977 : d'une part fin mai un écart moyen de $+460 \mathrm{~kg}$ (croissance plus précoce), d'autre part fin septembre un écart moyen de - $620 \mathrm{~kg}$ (dessèchement plus précoce).

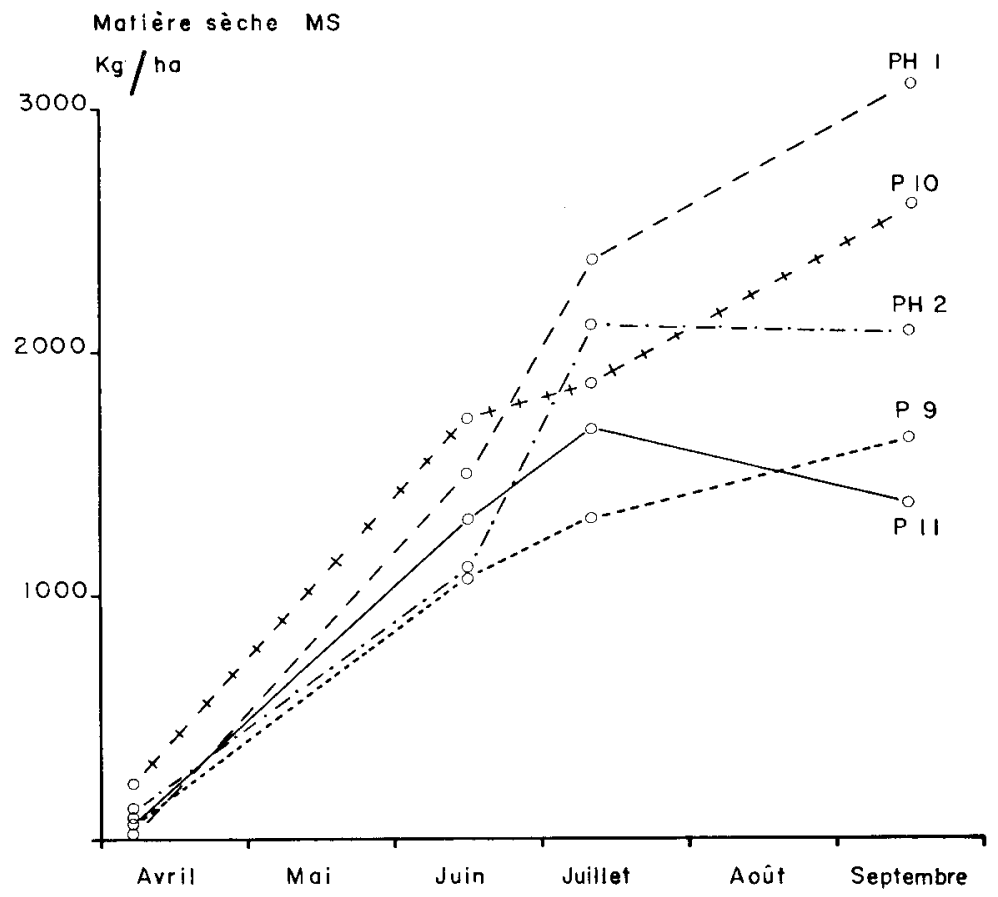

FIG. 2

Croissance pondérale cumulée de la molinie en 1977 (parties vivantes épigées).

Growth curves of above ground Molinia dry weight 1977.

PH1 : Hivernage forte charge.

P10 : Pâturé et fertilisé.

PH2 : Hivernage faible charge.

P9 : Pâturage estival.

P11 : Témoin non pâturé.

\subsection{Productivité de la pelouse (molinie ou molinie + avoine)}

D'autres sites ont été étudiés. Le tableau 1 fait l'inventaire des résultats globaux obtenus.

La production de la lande boisée humide ou mésophile humide paraît ainsi relativement faible et variée.

L'influence du rythme d'exploitation sur la production semble difficile à cerner pour l'instant. Elle apparaît soit positive, soit négative, selon les situations (parcelle, 
LANDES, ÉLEVAGE EN FORÊT

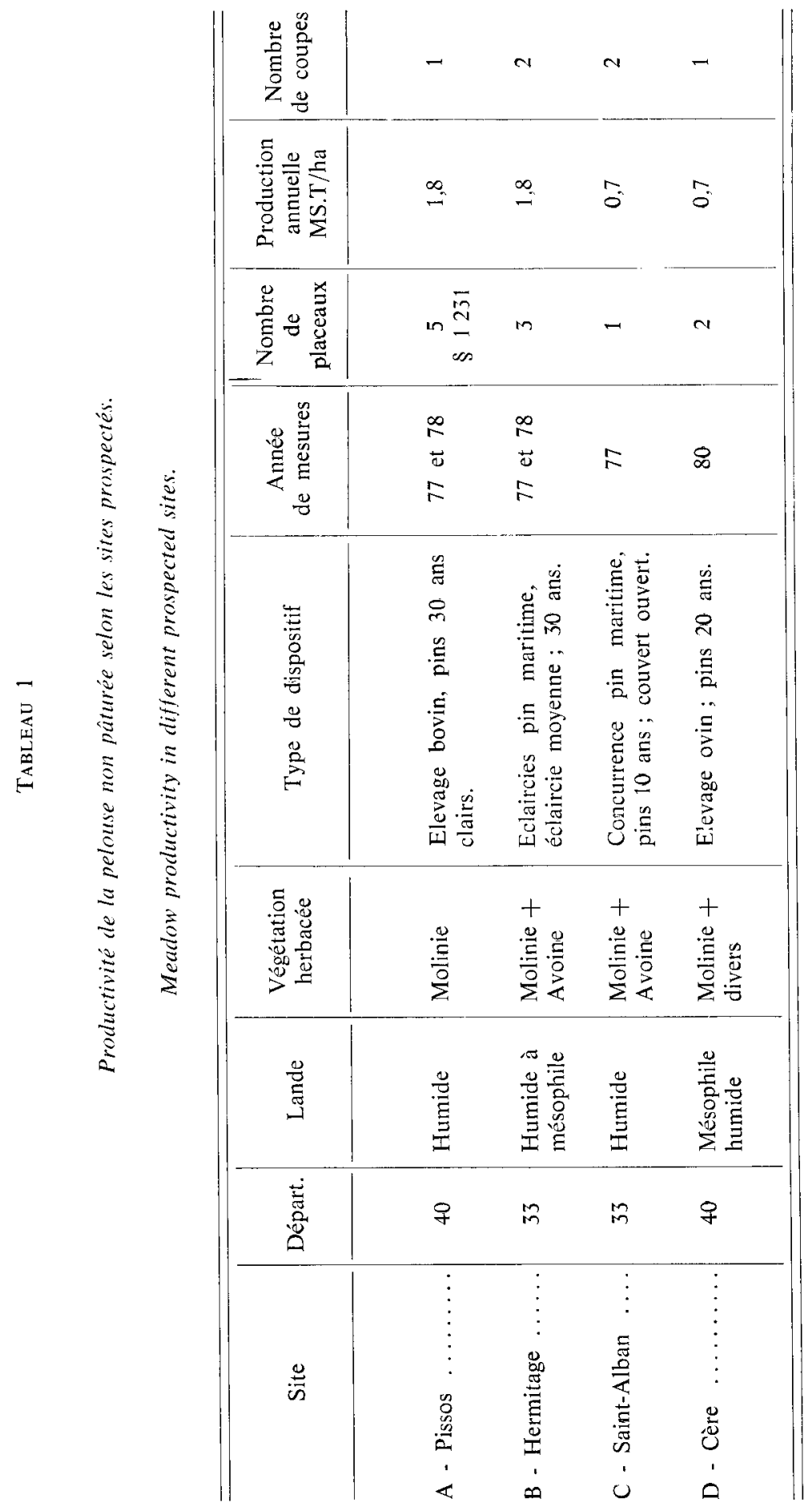


traitement...). Les résultats les plus nets semblent se situer au niveau interannuel : la production de molinie à l'année $\mathbf{n}$ est, d'une part favorisée par une coupe tardive à l'année $\mathrm{n}-1$, et d'autre part défavorisée par 3 coupes successives à l'année $\mathrm{n}-1$ (Bergeret H., 1980).

\subsection{Pelouse et peuplement de pins}

Les objectifs sont de :

- chiffrer l'influence de la densité du peuplement de pin sur la production de la pelouse en position de sous-bois;

- caractériser parallèlement les divers types de productivité de l'écosystème en fonction de la densité du peuplement de pins; on sait déjà que ce dernier paramètre a une action déterminante sur la production de bois (LEMOINE B. et al., 1976 ; Lemoine B., 1980).

On a travaillé pour ce faire dans deux dispositifs sylvicoles étudiant les effets sur le pin maritime, l'un de la densité de plantation, l'autre de l'intensité des éclaircies (cf. BERGERET H., 1980).

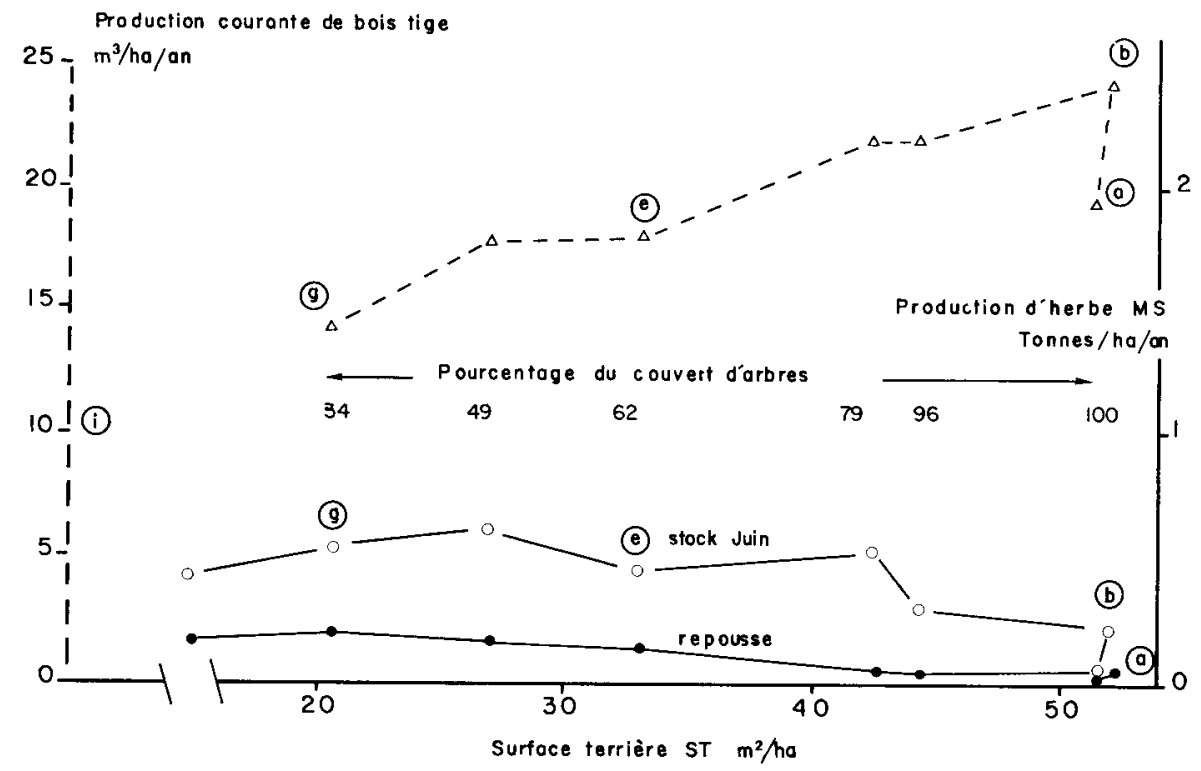

FIG. 3

Production de bois et d'herbe en fonction de la densité du peuplement forestier (surface terrière par hectare).

Dispositif à espacement variable de Saint-Alban (12 ans) 1977.

Wood and grass yields as a function of trees stand density (basal area per ha).

Variable spacing planting design (12 years) 1977.

Densités de plantation (nombre d'arbres à l'hectare) :
(a) 13050
(g) 876
(b) 8388
(i) 0 (plein découvert)
(e) 2152 


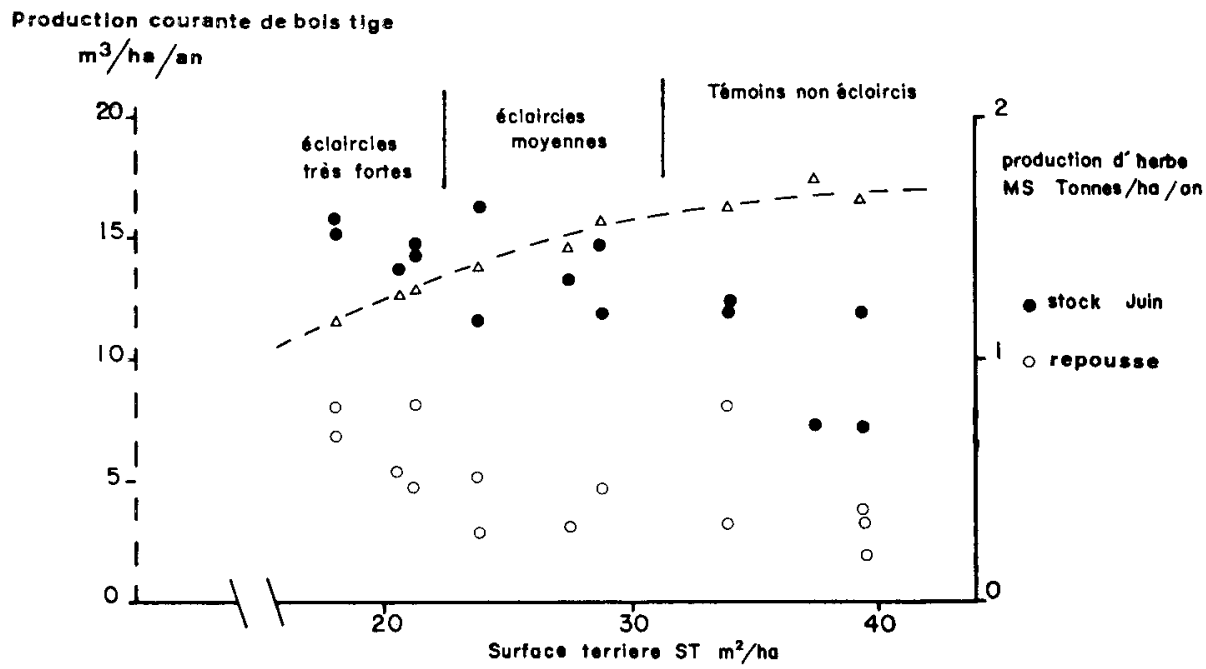

FIG. 3 bis

Production de bois et d'herbe en fonction de la densité du peuplement forestier (surface terrière par hectare).

Dispositif d'éclaircie de L'Hermitage (30 ans). 1977 et 1978.

Date de la dernière éclaircie : hiver 1975.

Wood and grass yields as a function of trees stand density (basal area per ha).

Thinning design (30 years). 1977 and 1978.

Date of the last thinning : Winter 1975.

Les figures 3 et 3 bis rassemblent l'essentiel des résultats. On confronte ici des tonnes de matière sèche d'herbe et des mètres cubes de bois-tige c'est-à-dire des unités de mesure différentes. On sait d'après les travaux de RANGER J. (1978) et de Cabanettes A. et al. (1978) sur deux espèces de pins qu'il faudrait appliquer à nos volumes produits une infradensité (bois à l'état sec) de l'ordre de 0,40 tonne $/ \mathrm{m}^{3}$. Cette remarque n'altère en rien la portée du raisonnement qui va suivre, d'autant plus que l'on devrait rajouter au bois-tige les autres compartiments de la biomasse aérienne du pin maritime, car les ordres de grandeur dégagés ici sont suffisamment tranchés.

L'expérience de densité de plantation représentée par la figure 3 (pins âgés de 12 ans) permet d'observer l'ensemble des états possibles du sous-bois herbacé en fonction des paramètres d'état du peuplement d'arbres, et notamment du pourcentage de couvert qui d'après les travaux de WARREN (1979) est une variable explicative de la production d'herbe meilleure que la surface terrière du peuplement d'arbres :

- sous les peuplements à couvert (projection des cimes) fermé ou peuplements du type II (Lemoine B., 1980) la production et la contribution spécifique de l'herbe sont nulles ou faibles; en ce qui concerne le peuplement d'arbres on remarque que, pour les plus fortes densités de plantation étudiées ici, l'effet, négatif, de la concurrence intraspécifique se porte, non seulement sur les accroissements courants, mais également sur la surface terrière c'est-à-dire sur la production cumulée (d'où l'aspect 
particulier de la courbe Production de bois - Surface terrière) ; pour les peuplements proche de ceux du type I, c'est-à-dire «serrés», la surface terrière devient une mesure criticable de la densité ;

- sous des peuplements à couvert de plus en plus ouvert ou peuplements du type III la production et la contribution spécifique de l'herbe augmentent puis tendent vers certaines limites bien avant l'état de plein découvert; on notera que la plus forte densité laissée à cet âge par certains sylviculteurs, soit 2500 arbres/ha, doit permettre au sous-bois d'atteindre une production peu éloignée de son potentiel.

Parallèlement la production du peuplement d'arbres, qui est maximum lorsque le couvert est tout juste fermé, diminue de façon très spectaculaire (jusqu'à $15 \mathrm{~m}^{3} /$ ha/an) lorsque la densité des arbres diminue; il en est manifestement de même pour la production (épigée) du système végétal entier à deux strates.

Les résultats de l'expérience d'éclaircies de pins maritimes âgés de 30 ans (figure 3 bis) permettent d'arriver à des conclusions similaires, bien que, d'une part on doive se contenter de la surface terrière comme paramètre de densité, que d'autre part la fourchette de densités soit moins grande et qu'encore l'équilibre de productivité des 2 strates soit différent et moins à l'avantage des arbres.

La conclusion, provisoire, de cette partie de l'étude est que l'augmentation de production d'herbe est loin de compenser, dans ces expériences, la diminution de production du peuplement d'arbres quand on diminue la densité de ce dernier (par des densités de plantation plus faibles ou des éclaircies plus fortes).

\subsection{Ambiance forestière}

Le problème est de caractériser les conditions bioclimatologiques du fonctionnement de la pelouse en situation de sous-bois. Certains paramètres relativement faciles à apprécier sont ainsi mesurés simultanément dans la parcelle boisée et dans une parcelle non boisée de référence (pépinière au sein du massif boisé de l'Hermitage). Ces paramètres sont les suivants :

- la température 0 et l'humidité relative $U$ de l'air sous abri météorologique ;

- le chemin parcouru par le vent à 2 mètres au-dessus du sol pendant une semaine, d'où sa vitesse moyenne $\mathrm{V}$ en $\mathrm{m} / \mathrm{sec}$;

- la perméabilité $P_{R g}$ du couvert d'arbres au rayonnement global $\mathrm{Rg}$ obtenue soit par des mesures comparatives à la pile de Moll, soit par des photographies hémisphériques; les mesures de rayonnement net $\mathrm{Rn}$ n'ont pu être effectuées avant cette année par manque d'équipement.

La mesure de ces paramètres se justifie sur le plan biologique de la façon suivante :

$\theta$ : son évolution annuelle est à relier à la saison de végétation pour la pelouse au niveau du mésoclimat;

$\mathrm{Rg}$ : apprécie les conditions de photosynthèse (cf. $\$ 2.35$ ) ;

$\theta$, $\mathrm{Rn}$ (ou son approximation par $\mathrm{Rg}$ ), $\mathrm{U}$ et $\mathrm{V}$ permettent d'approcher par un bilan énergétique les conditions de fonctionnement hydrique de la plante; tous les paramètres mesurés seront donc utilisés synthétiquement dans la formule de Penmann (Brochet P., Gerbier N., 1972). 
Les résultats obtenus sont les suivants :

a) la température $\theta$ de l'air n'est pas affectée par le couvert;

b) la perméabilité relative au rayonnement global soit $P_{\mathrm{Iig}}$ : l'exploitation de photographies hémisphériques, prises en avril, par un programme Fortran écrit pour ordinateur C.I.I. 10070 (DuCreY M., 1975) permet de différencier les traitements sylvicoles pour 2 types de temps, ciel couvert et ciel clair et dans ce dernier cas pour chacune des 4 saisons (figure 4 ) : les courbes $P_{\mathrm{kg}}=\mathrm{f}$ (date) par ciel clair et pour les différents traitements se déduisent les unes des autres par affinité orthogonale par rapport à l'axe des dates; la perméabilité par ciel couvert est très voisine de la perméabilité moyenne pendant la saison de végétation; la surface terrière n'est pas un très bon estimateur de $P_{\mathrm{ig}}$ quand on envisage des parcelles très différentes (parcelles $\mathrm{S} \mathrm{A}$ et $\mathrm{P} \mathrm{J}$ );

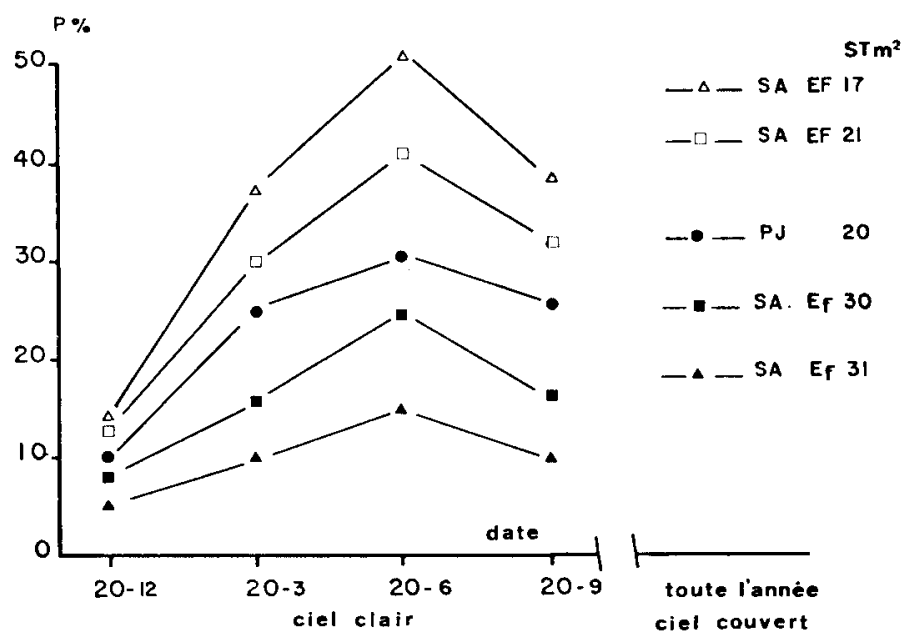

FIG. 4

Perméabilité relative $P$ p. 100 au rayonnement global en fonction du type de temps et de la saison. Exploitation des photographies hémisphériques.

Relative permeability to global radiation as a function of wheather situation and season. Use of hemispheric pictures.

SA : Dispositif d'éclaircies de Saint-Alban (30 ans).

PJ : Peuplement de Petit-Jarry (30 ans).

EF : Eclaircie forte.

Ef: Eclaircie faible.

ST : Surface terrière.

c) l'humidité relative $U$ n'est pas affectée par le couvert;

d) le vent $\mathrm{V}$ est modifié par le couvert selon une loi très simple : pour l'éclaircie moyenne (figure 5) la relation entre $V_{2}$ sous couvert et $V_{1}$ à découvert est la suivante :

$$
\mathrm{V}_{2}=0,35 \cdot \mathrm{V}_{1}
$$




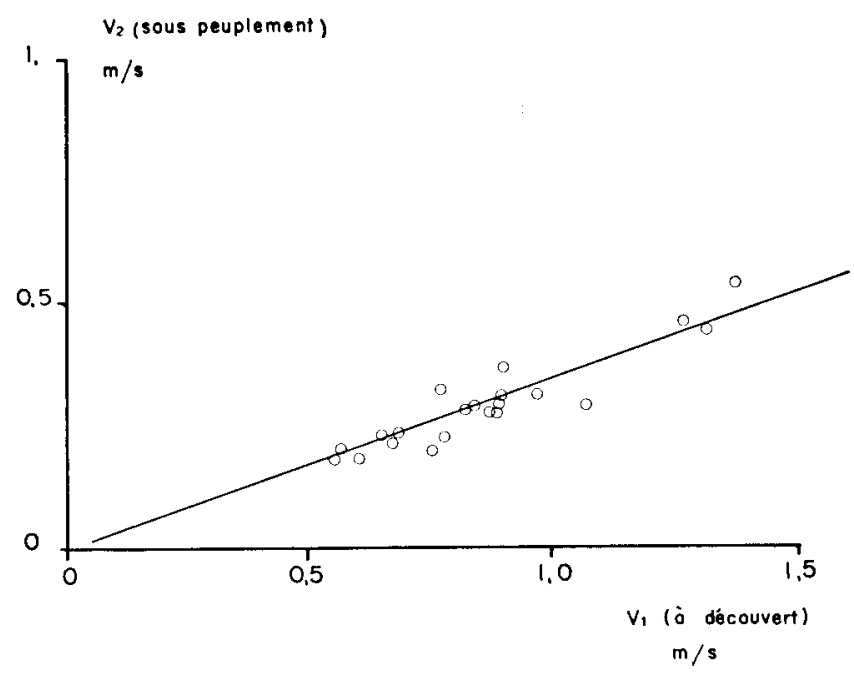

Fig. 5

Comparaison des vitesses de vent sous forêt et à découvert.

$V_{1}$ et $V_{2}=$ chemin moyen parcouru par le vent à 2 mètres au-dessus du sol. Eclaircie moyenne à 30 ans (surface terrière : $29 \mathrm{~m}^{2}$ ).

Comparison of the average wind velocity in the underwood and in the open field.

Middle thinning (Basal area $=30 \mathrm{~m}^{3}$ ).

$V_{1}$ and $V_{2}$ represent the distance covered by the wind at $2 \mathrm{~m}$ from the soil surface.

Farsworth (1979) a procédé à une étude dans un peuplement de pins insignis en Nouvelle-Zélande. La finalité de son travail est la même que la nôtre («Farm Forestry »); il étudie la vitesse moyenne mensuelle, à $2 \mathrm{~m}$ du sol (et à $1 \mathrm{~m}$ ), par rapport à une référence extérieure (Open site) depuis l'installation du peuplement jusqu'à l'âge de 6 ans : de 2 à 6 ans le rapport $V_{2} / V_{1}$ évolue de la façon suivante : 70 p. 100,60 p. 100,6 p. 100 et 29 p. 100 : cette dernière remontée du rapport en question est due à l'accélération du relèvement du couvert par l'élagage artificiel ; les conditions de son étude (pins insignis à 6 ans) et celles de la nôtre (pin maritime à 28 ans) sont alors relativement analogues puisque le vent ne rencontre à $2 \mathrm{~m}$ sous couvert que le seul obstacle des troncs.

Nous formulons les remarques suivantes :

- Pendant la période préprintanière la faiblesse du rayonnement global extérieur est accentuée sous forêt par les très faibles valeurs de la perméabilité relative ; les températures $\theta_{\mathrm{m}}$ minima sous abri ne sont pas modifiées par le couvert d'arbres; pour savoir s'il existe des différences notables de longueur de végêtation et de ses conditions climatiques — au niveau du mésoclimat - il faudrait, d'une part procéder à des mesures sur le végétal (molinie par exemple) dans les deux situations sous-couvert et de plein découvert, d'autre part mesurer d'autres paramètres, principalement la température du sol et le rayonnement net. 
- La valeur de l'ETP s'obtient par la formule de Penmann en faisant la somme de deux termes, l'un radiatif ou $\varphi(\mathrm{Rn})$, l'autre advectif ou $\varphi(\mathrm{Ea})$, soit :

$$
\mathrm{ETP}_{\mathrm{mm} .}=\varphi(\mathrm{Rn})+\varphi(\mathrm{Ea})
$$

Au niveau global (hypothèse d'une seule strate évaporante) et au plan local on a pu établir (travail non publié) qu'ici le terme radiatif représente en moyenne 75 p. 100 de l'ETP. Le terme advectif se détaille ainsi :

$$
\varphi(\mathrm{Ea})=\frac{0,26}{1+\frac{\mathrm{F}^{\prime} \mathrm{T}}{\gamma}} \cdot\left(\mathrm{e}_{w}-\mathrm{e}\right) \cdot(1+0,54 . \mathrm{V})
$$

où $\mathbf{F}_{T}^{\prime}=$ pente de la courbe de la pression de vapeur saturante en fonction de la température, $\gamma=$ constante psychrométrique, $\left(\mathrm{e}_{\mathrm{w}}-\mathrm{e}\right)=$ déficit de pression de vapeur saturante, $\mathrm{V}=$ vitesse du vent. Compte tenu de ce qui a été dit plus haut, la modification de $\varphi(\mathrm{Ea})$ sous couvert par rapport au découvert est due à la seule modification du vent : $\varphi($ Ea) subit ainsi une réduction moyenne de seulement $20 \mathrm{p}$. cent. Observant que les réductions de $\mathrm{Rg}$ sont bien plus importantes, on peut penser que sous couvert le terme advectif $\varphi(E a)$ devient du même ordre de grandeur que le terme radiatif $\varphi(\mathbf{R n})$.

\subsection{Relation herbe-lumière}

On a procédé à deux types de travaux :

- d'une part sur la flore implantée; cette démarche est à mettre en relation avec l'éventualité de l'établissement de prairies artificielles;

- d'autre part, sur la flore spontanée; cette autre démarche a été motivée par les résultats des essais d'implantation de graminées fourragères (investissements exigés et résultats obtenus; cf. $\S 2.2$ ).

\section{a) La fétuque introduite}

(cf. Galley, 1977 ; Allard, 1979 et Commeres A., 1980)

Les phénomènes de compétition inter-strates végétales en forêt s'exercent sur les facteurs de productions et en particulier la lumière, l'eau, les éléments minéraux.

Dans le cas du sous-bois landais, la lumière joue-t-elle le rôle de facteur limitant primaire de la croissance de l'herbe et modifie-t-elle sa valeur nutritive?

Pour essayer de répondre à cette question, une expérimentation a été entreprise de 1976 à 1979 sur une parcelle mise à notre disposition par la C.A.R.A.

Protocole : afin d'éviter les interactions et sur les conseils de DE MonTARD (I.N.R.A., Clermont) une expérimentation de Fétuque semée sur une bande pare-feu non pâturée à Pissos en ombrage artificiel a été mise sur pied. 
Quatre niveaux d'ombrage avec quatre répétitions ont été comparés sur petites parcelles $\left(4 \mathrm{~m}^{2}\right)$ grâce à des grillages plastiques placés à $50 \mathrm{~cm}$ du sol et à mailles différentes :
100 p. 100 de l'éclairement;
60 p. 100 de l'éclairement;
45 p. 100 de l'éclairement;
25 p. 100 de l'éclairement.

Trois à quatre coupes ont été effectuées chaque année pendant 4 ans, en fonction de la pousse de l'herbe. A chaque coupe, le rendement était mesuré et un prélèvement pour analyse effectué.

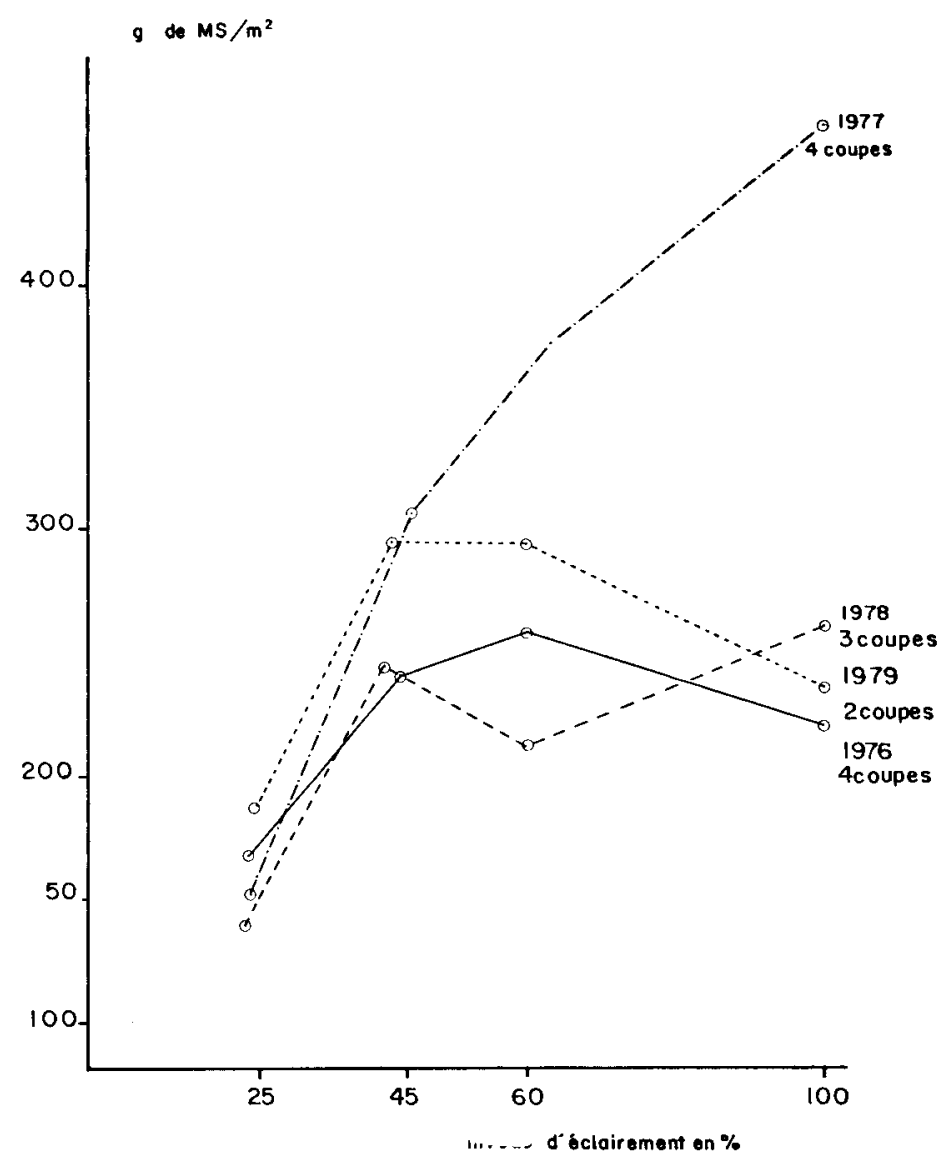

FIG. 6

Influence de l'éclairement sur le rendement de la fétuque à Pissos (ombrages artificiels). Effect of light (p. 100 of radiation) upon Fescue yield in Pissos (artificial shade). 


\section{Résultats :} climatiques :

- quantitatifs (figure 6) : les résultats sont très variables selon les conditions

- en année sèche (1976 et 1978) la lumière ne joue pas le rôle de facteur limitant : au-delà d'un taux d'éclairement de 40 à 60 p. 100 les rendements n'augmentent plus. L'eau est alors facteur limitant primaire ;

- en année humide, le facteur limitant n'est plus l'eau mais la lumière (1977);

- quelle que soit l'année en dessous d'un seuil d'éclairement voisin de 40 p. 100 la production est limitée par manque de lumière.

Dans un peuplement de pins bien conduit la perméabilité relative est, à partir de 20 ans, inférieure ou égale à ce seuil (LEMoine, communication personnelle). point.

Les expériences faites sur la flore naturelle doivent permettre de préciser ce

- qualitatifs (tabl. 2) : les analyses effectuées ne révèlent pas de modification importante de la composition de la Fétuque sous l'influence de l'intensité de l'éclairement.

\section{TABLEAU 2}

Valeur nutritive de la fétuque en fonction de l'intensité de l'éclairement reçu (in Allard, 1979).

Radiation effect upon Fescue food value.

\begin{tabular}{|c|c|c|c|c|}
\hline Traitement & A & B & $\mathrm{C}$ & $\mathrm{D}$ \\
\hline Taux d'éclairement $\ldots \ldots \ldots \ldots \ldots \ldots \ldots$ & $100 \%$ & $60 \%$ & $45 \%$ & $25 \%$ \\
\hline$\%$ de matière sèche $(\mathrm{MS}) \ldots \ldots \ldots \ldots \ldots \ldots$ & 28,16 & 22,36 & 20,10 & 17,91 \\
\hline $\begin{array}{l}\text { Coefficicnt d'utilisation digestive (C.U.D.) de la } \\
\text { matière organique } \ldots \ldots \ldots \ldots \ldots \ldots \ldots \ldots \ldots \ldots\end{array}$ & 68,08 & 68,02 & 67,52 & 67,41 \\
\hline Matière minérale $\%$ de $\mathrm{MS} \ldots \ldots \ldots \ldots \ldots$ & 6,41 & 6,78 & 7,51 & 9,22 \\
\hline Matière azotée totale $\%$ de $\mathrm{MS} \ldots \ldots \ldots \ldots \ldots$ & 13,73 & 13,82 & 14,93 & 15,91 \\
\hline Cellulose brute $\%$ de MS $\ldots \ldots \ldots \ldots \ldots \ldots$ & 25,95 & 26,47 & 27,19 & 26,81 \\
\hline Unités fourragères $/ \mathrm{kg}$ de $\mathrm{MS} \ldots \ldots \ldots \ldots \ldots$ & 0,69 & 0,68 & 0,66 & 0,65 \\
\hline 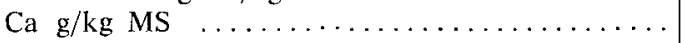 & 5,31 & 4,78 & 4,68 & 4,86 \\
\hline $\mathrm{P} \quad \mathrm{g} / \mathrm{kg} \mathrm{MS} \ldots$ & 4,29 & 4,39 & 4,14 & 5,21 \\
\hline $\mathrm{Fe} \mathrm{mg} / \mathrm{kg}$ de $\mathrm{MS}$ & 141,62 & 160,32 & 166,17 & 202,65 \\
\hline $\mathrm{Mn} \mathrm{mg/kg} \mathrm{de} \mathrm{MS}$ & 252,56 & 241,49 & 282,76 & 239,69 \\
\hline $\mathrm{Zn} \mathrm{mg/kg} \mathrm{de} \mathrm{MS}$ & 36,36 & 34,87 & 48,02 & 53,26 \\
\hline
\end{tabular}

Ne sont inclus dans ce tableau que les résultats moyens obtenus pour chaque traitement sur l'ensemble des années : $1976: 4$ coupes; $1977: 4$ coupes $; 1978: 2$ coupes.

On peut noter cependant, avec l'augmentation de l'intensité lumineuse, une tendance à : 
- l'augmentation de la teneur en matière sèche, de la digestibilité et donc de la valeur énergétique ;

- la diminution de la teneur en matières minérales, en matière azotée totale et cellulose brute.

En définitive, l'intensité lumineuse ne paraît guère modifier la valeur nutritive de la Fétuque. Par contre, elle joue sur le rendement : dans tous les cas lorsque moins de 40 p. 100 environ de la lumière solaire arrive à la plante, et seulement en année humide pour des seuils plus élevés.

\section{b) La flore naturelle}

Molinie et Avoine de Thore (cf. Bergeret H., 1980)

Il s'agit ici d'une première recherche effectuée in situ dans le dispositif d'éclaircie sur des pins de 30 ans dont il est question au $\$ 2.33$.

L'explication des variations de la production d'herbe par les variations d'état du peuplement forestier peut se faire à l'aide d'un des 3 paramètres suivants :

- la surface terrière ;

- le pourcentage de couvert;

- la lumière parvenant au sol.

Il paraît logique, en site humide, d'approcher au mieux le facteur limitant de la pousse de l'herbe qui est la lumière; en conséquence on préférera utiliser le $2^{\circ}$ paramètre plutôt que le $1^{\mathrm{er}}$, le $3^{\circ}$ plutôt que le $2^{\circ}$. Cette observation est à mettre en balance avec les contraintes de l'expérimentateur mais on observera déjà que :

- le $2^{\text {e }}$ paramètre explique mieux la pousse de l'herbe que le $1^{\text {er }}$ paramètre (cf. § 2.33);

- le $3^{\text {e }}$ paramètre n'est qu'imparfaitement expliqué par le $1^{\text {er }}$ paramètre (cf. $\S 2.34$ ),

et qu'il revient au dendrométricien et au bioclimatologiste forestier d'expliquer les divergences entre ces 3 paramètres.

Sur les 9 placeaux étudiés $(3$ blocs, de mésophile humide à humide $\times 3$ traitements : témoin non éclairci, éclaircie moyenne et éclaircie très forte) on dispose, en plus de la production et de la contribution spécifique de l'herbe mesurées en 1978, du rayonnement global relatif $\mathrm{P}_{\mathrm{lag}}$ mesuré en mai à la pile de Mollo.

La relation production annuelle 1978 - éclairement relatif est, après examen graphique, justiciable d'un ajustement curvilinéaire. Nous choisissons provisoirement le modèle le plus simple, celui de l'hyperbole équilatère, d'où l'équation :

$$
\Sigma\left(\frac{\Delta \mathrm{Ms}}{\Delta \mathrm{t}}\right)=3,244-0,477 / \mathrm{P}_{\mathrm{Igg}}
$$

avec une corrélation de :

$$
\mathbf{r}=-0,900 * * *(\mathrm{dl}=7)
$$


L'utilisation de cette équation en dehors de son domaine de validité $\left(0,22 \leqslant \mathbf{P}_{\mathrm{Rg}}\right.$ $\leqslant 0,43$ ) fournit certains renseignements d'ordre théorique que nous serons en mesure de confirmer ou d’infirmer expérimentalement :

- la production herbacée à découvert $\left(\mathrm{P}_{\mathrm{Rk}}=1\right)$ serait dans ce site de 2,8 tonnes de MS/ha/an;

- la production sannulerait pour un rayonnement global relatif de 15 p. 100.

L̈intensité dı phénomène d’ordre photosynthétique est peut-être, à travers le coefficient de régression $b=-0,477$ de l'équation (2), surestimée : il existe en effet, d'une part entre la contribution spécifique (C.S.s.) et le rayonnement global relatif $\left(\mathrm{P}_{\mathrm{RR}}\right)$ une corrélation simple, d'autre part entre la production $\left(\frac{\Delta \mathrm{Ms}}{\Delta \mathrm{t}}\right)$ et la contribution spécifique une corrélation partielle, ces deux corrélations étant très peu éloignées du seuil de signification statistique de 5 p. 100.

Par ailleurs la mise en évidence d'un éventuel effet de coopération d'un couvert très léger pour lequel nous manquons ici de données, exigerait l'emploi d'un modèle parabolique plutôt qu'hyperbolique (cf. \$2.37).

La contribution spécifique de la pelouse, qui peut d'ailleurs en l'absence de ligneux bas sassimiler à un taux d’occupation du sol, doit donc être considérée comme un paramètre non négligeable de la productivité primaire. Dans le cadre de l'expérimentation sylvicole dont il est question ici ces variations qui seraient, soit commandées par les variations d'éclairement, soit de caractère aléatoire, influenceraient la productivité.

\subsection{Modélisation de la production de la pelouse}

Lobjectif est de mettre sur pied un modèle prédictif de la production de la $\Delta \mathrm{MS}$

biomasse herbacée, soit des $\frac{\Delta M S}{\Delta t}$, en fonction des variations habituelles du climat à l'intérieur de l"année.

Différents auteurs (RoBElin M., 1958 et 1969 ; Bouchet P., 1964 et MIQUEUX M., 1979 ; JEANNIN B. et al., 1979) ont réussi à mettre en évidence une relation linéaire entre production de matière sèche et consommation en eau pour une demande climatique donnée.

On peut utiliser également un modèle faisant jouer l'énergie et le déficit hydrique, voire la température (DE MONTARD F., 1981); c'est de ce dernier dont nous nous sommes inspirés.

Dans le cas présent la production de la végétation étudiée, pelouse en sous-bois, est conditionnée par une strate arborée. Celle-ci joue un effet sans conteste sur les conditions climatiques déterminant directement la production de la strate herbacée; d'une part en raison du phénomène d'interception des pluies, d'autre part en raison de la réduction du rayonnement parvenant au sol.

Dans ces conditions il devient logique d'appliquer à la pelouse une formule d'évapotranspiration incluant les paramètres de modifications climatiques déterminés par la strate arborée. Il devient ainsi possible d'utiliser un modèle valable aussi bien en position de sous-bois qu'en plein découvert. 
On cherche à expliquer statistiquement les variations des $\frac{\Delta \mathrm{MS}}{\Delta \mathrm{t}}$ en fonction de variables exprimant :

- soit la consommation en eau, ETR ;

- soit la combinaison de l'énergie reçue, ETP, et du déficit d'alimentation en eau exprimée en valeur absolue, ETP - ETR, ou en valeur relative (ETP - ETR)/ ETP.

Compte tenu des contraintes et des données existantes nous nous sommes arrêtés à l'emploi de la formule de Turc (1961) :

$$
\mathrm{ETP}=0,013 \cdot \mathrm{n} \cdot \frac{\mathrm{t}}{\mathrm{t}+15} \cdot\left(\mathrm{Rg}^{\prime}+50\right)
$$

où $\mathrm{n}=$ nombre de jours de la période considérée ;

$\mathrm{t}=$ température normale ;

$\mathrm{Rg}^{\prime}=$ rayonnement global sous couvert $=\mathrm{Rg} \cdot \mathrm{P}_{\mathrm{Rg}} ;$

$\mathrm{P}_{\mathrm{Rg}}=$ éclairement relatif (cf. $\S 2.2$ ).

TABLEAU

Explication des accroissements saisonniers en biomasse de la pelouse

$$
\frac{\Delta M S}{\Delta t}(\mathrm{~kg} / \text { jour }) \text { dans } 2 \text { sites en 1977-1978. }
$$

\begin{tabular}{|c|c|c|c|c|c|}
\hline \multirow{3}{*}{$\begin{array}{c}\mathrm{N}^{\circ} \\
\text { équation }\end{array}$} & \multirow{3}{*}{ Site } & \multirow{3}{*}{ Modalités } & \multirow{3}{*}{ Constante } & \multicolumn{2}{|c|}{ Varia } \\
\hline & & & & $\Delta \mathrm{ETP}$ & $\triangle$ ETRO \\
\hline & & & & $\Delta \mathrm{t}$ & $\Delta t$ \\
\hline I & Pissos (A) & Témoin non pâturé & $-41,27$ & & $42,18^{*}$ \\
\hline II & Pissos (A) & Témoin non pâturé & $-18,08$ & & \\
\hline III & Hermitage (B) & 3 blocs $\times 3$ éclaircies & $-0,37$ & & $10,49 * * *$ \\
\hline IV & Hermitage (B) & 3 blocs $\times 3$ éclaircies & 2,13 & & \\
\hline V & Pissos & Témoin non pâturé & $-15,96$ & $28,39 *$ & \\
\hline VI & Pissos & Témoin non pâturé & $-7,51$ & $24,82^{*}$ & \\
\hline VII & Hermitage (B) & 3 blocs $\times 3$ éclaircies & 3,66 & $7,81 * *$ & \\
\hline VIII & Hermitage (B) & 3 blocs $\times 3$ éclaircies & 8,45 & $5,36^{* *}$ & \\
\hline IX & Pissos & $\begin{array}{l}\text { Parcelles témoin } \\
\text { et pâturées }\end{array}$ & $-26,26$ & $38,74^{* * *}$ & \\
\hline
\end{tabular}

Régressions multiples en fonction de variables climatiques et de caractères du système d'élevage. 
Dans l'établissement du bilan hydrique déterminant l'ETR pour une période définie, la quantité de pluie parvenant à la strate herbacée a été calculée selon la formule d'Aussenac définie pour le pin sylvestre (1968) :

$$
\mathscr{E}_{N}(\mathrm{Y}+1)=\mathrm{b}_{0}+\mathrm{b}_{1} \cdot \mathrm{P}_{\mathrm{Rg}}+\mathrm{b}_{2} \cdot \mathrm{X}+\mathrm{b}_{3} \cdot \mathrm{X}^{2}
$$

où $\mathrm{Y}=$ précipitations arrivant au sol pendant la période considérée;

$\mathbf{P}_{\mathbf{R g}}=$ éclairement relatif ;

$\mathrm{X}=$ pluies incidentes (au-dessus du peuplement d'arbres).

Pour la réserve utile, $R_{\mathrm{u}}$, à affecter à la strate herbacée, 2 hypothèses ont été comparées :

- calcul décadaire avec écrêtage des pluies journalières supérieures à $20 \mathrm{~mm}$ et sommation sur 10 jours, sans comptabilisation d'aucune réserve en eau au sol : ETRO ;

- calcul journalier avec réserve en eau du sol de $20 \mathrm{~mm}: \mathbf{E T R}_{\mathbf{2 0}}$.

Le tableau 3 récapitule les principaux essais d'ajustement effectués. On peut formuler les deux remarques suivantes :

Seasonal meadow biomass increments, $\frac{\Delta M S}{\Delta t}(\mathrm{~kg} / \mathrm{ha} /$ day $)$

in 2 sites during 1977 and 1978 .

\begin{tabular}{|c|c|c|c|c|c|c|}
\hline \multicolumn{3}{|c|}{ lat } & \multicolumn{2}{|c|}{ Variable élevage } & \multirow{4}{*}{$\mathrm{dl}$} & \multirow{4}{*}{$\begin{array}{c}\text { Corrélation } \\
\text { globale }\end{array}$} \\
\hline$\Delta \mathrm{ETR}_{20}$ & $\frac{\Delta \mathrm{ETP}}{\Delta t}$ & 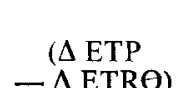 & $\mathrm{J}$ & $(I)^{2}$ & & \\
\hline$\Delta \mathrm{t}$ & $\triangle \mathrm{ETR} \theta$ & 4 & $\mathrm{~T}$ & $(\mathrm{~T})$ & & \\
\hline & $\Delta t$ & 851 & & & & \\
\hline \multirow{10}{*}{$18,54 \mathrm{NS}$} & \multirow{10}{*}{$-45,69 *$} & \multirow{10}{*}{$-20,39 * * *$} & & & 5 & 0,7315 \\
\hline & & & & & 5 & 0,395 \\
\hline & & & & & 28 & 0,580 \\
\hline & & & & & 28 & 0,441 \\
\hline & & & & & 4 & 0,939 \\
\hline & & & & & 4 & 0,959 \\
\hline & & & & & 27 & 0,659 \\
\hline & & & & & 27 & 0,726 \\
\hline & & & 1,19 NS & $-0,035$ & \multirow[t]{2}{*}{22} & \multirow[t]{2}{*}{0,857} \\
\hline & & & & & & \\
\hline
\end{tabular}


- pour le calcul de l'ETR, l'hypothèse réserve utile $R_{11}=0 \mathrm{~mm}$ s'avère plus performante que l'hypothèse $R_{u}=20 \mathrm{~mm}$ (cf. équations I, II, III et IV) ceci est vrai pour chacun des 2 sites étudiés et aussi pour des équations du type V, VI, VII et VIII ; ceci permet d'avancer qu'il existe au niveau des racines des graminées du sousbois un phénomène d'interception des flux d'eau occasionnés par les pluies;

- le modèle le plus performant dans les 2 sites est celui qui utilise l'énergie reçue et le déficit relatif (cf. équations VI et VIII); il revêt la forme suivante :

$$
\frac{\Delta \mathrm{MS}}{\Delta \mathrm{t}}=\mathrm{a}_{0}+\mathrm{a}_{1} \cdot \frac{\Delta \mathrm{ETP}}{\Delta \mathrm{t}}+\mathrm{a}_{2} \cdot(\Delta \mathrm{ETP}-\Delta \mathrm{ETR} \theta) / \Delta \mathrm{ETP}
$$

Un résultat encourageant est à signaler dans l'optique d'une généralisation de ce type de modèle: par rapport aux travaux effectués pour les prairies, non boisées, des montagnes d'Auvergne (DE MONTARD F., 1981) on constate une bonne convergence, dès que l'échantillon est assez important (Site B), d'une part entre les coefficients de corrélation globaux obtenus — Landes : 0,726; Auvergne : 0,698 —, d'autre part entre les coefficients de régression $a_{.2}$, qui chiffrent l'effet du déficit hydrique pendant la période de croissance mesurée - Landes : - 20,39; Auvergne : - 18,2 —.

\subsection{Ecologie expérimentale de la molinie}

L'objectif est de déterminer l'influence de la lumière et de l'eau (par défaut et par excès) sur la production de la molinie, et par-là même de confirmer ou d'expliquer les résultats trouvés sur le terrain; l'objectif est aussi de vérifier le bienfondé de la modélisation.

On a implanté des populations de molinie, par prélèvement d'éclats, dans des bacs de végétation.

Le dispositif statistique combine différents degrés d'ombrage par filets plastiques horizontaux (éclairements théoriques de 100 p. 100,50 p. 100,25 p. 100 et 12,5 p. 100), et de régime hydrique (évapotranspiration maximale soit ETM, $\frac{\text { ETM }}{2}$, nappe maintenue à $-30 \mathrm{~cm}$ soit $\mathrm{N}_{30}$ ou à $-20 \mathrm{~cm}$ soit $\mathrm{N}_{20}$ );3 répétitions sont installées sous serre plastique et 2 à l'extérieur. Les résultats d'accroissements de $\triangle \mathrm{MS}$

biomasse, soit $\longrightarrow$, sont obtenus par des coupes échelonnées tous les 45 jours. $\Delta \mathrm{t}$

Afin de pouvoir obtenir par voie de régression un modèle de réponse à la lumière commun aux données sous serre et aux données extérieures on a procédé (le 30-9-1980) à une mesure d'éclairement relatif au luxmètre soit $P_{1 u x}$. Les consommations en eau ont été mesurées sous serre y compris pour un témoin «terre nue ».

Les résultats observés au terme d'une première année d'expérimentation sont les suivants :

- concernant l'effet de l'excès d'eau on peut déjà conclure à une absence d'effet des deux régimes d'hydromorphie $\left(\mathrm{N}_{30}\right.$ et $\mathrm{N}_{20}$ ) par comparaison avec le régime optimal d'alimentation en eau (ETM), et ceci quel que soit le niveau d'éclairement;

- concernant l'effet de l'éclairement en conditions hydriques optimales (ETM) : Les données de production annuelle $\left(\Sigma \frac{\Delta \mathrm{MS}}{\Delta \mathrm{t}}\right)$ s'ajustent aux données d'éclaire- 


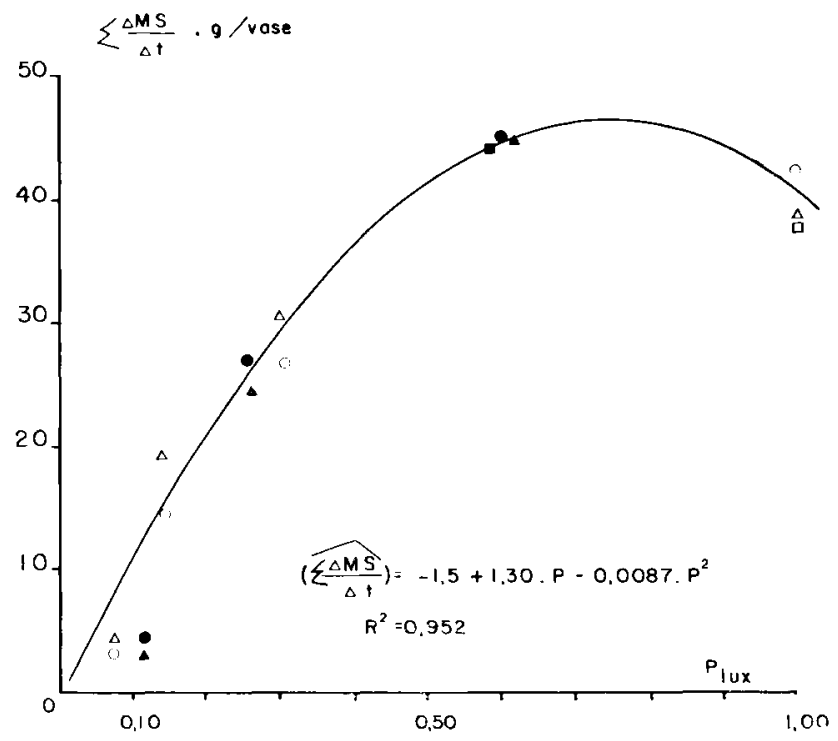

FIG. 7

Production annuelle (1980) de la molinie, en vases de végétation, en fonction de l'éclairement relatif. Régimes moyens et humides.

Annual yield of Molinia in pots as a function of relative radiation. Average and humid water supplies.

\begin{tabular}{|c|c|c|}
\hline & E T M & $\mathrm{N}_{20}$ \\
\hline Extérieur $\ldots \ldots \ldots$ & - & $\triangle$ \\
\hline$\ldots \ldots \ldots \ldots$ & 9 & $\Delta$ \\
\hline
\end{tabular}

ment $\left(\mathrm{P}_{\text {lux }}\right.$ ) selon un modèle parabolique (figure 7 ). Les données de production périodique ( $\Delta \mathrm{MS}$ pour $\Delta \mathrm{t}=45$ jours) donnent naissance à des courbes expérimentales de niveaux et de formes variés (figure 8) : les classements, par période, des traitements (éclairements relatifs P) par ordre de production décroissante sont ainsi :

- au printemps $\left(1^{\text {re }}\right.$ et $2^{\circ}$ coupes $)$ :

$$
P_{0,60}, P_{1,011}=P_{0,26} \quad P_{1,311}, P_{0,14}
$$

- à l'été $\left(3^{r}\right.$ coupe) :

$$
P_{0,30}=P_{0,611}=P_{1,010} \cdot P_{1,26} \cdot P_{0.14}
$$

- à l'automne $\left(4^{\mathrm{e}}\right.$ coupe $)$ :

$$
P_{1,00}=P_{0,60} \cdots P_{0.30} \quad P_{1,26} \cdots P_{0.14}
$$

On constate donc qu'un ombrage modéré compense l'effet négatif de rayonnements extérieurs saisonniers élevés :

- concernant l'effet d'un déficit hydrique $\left(\frac{\mathrm{ETM}}{2}\right)$ et son interaction avec l'éclairement : deux points sont à considérer (cf. figure 8) : 


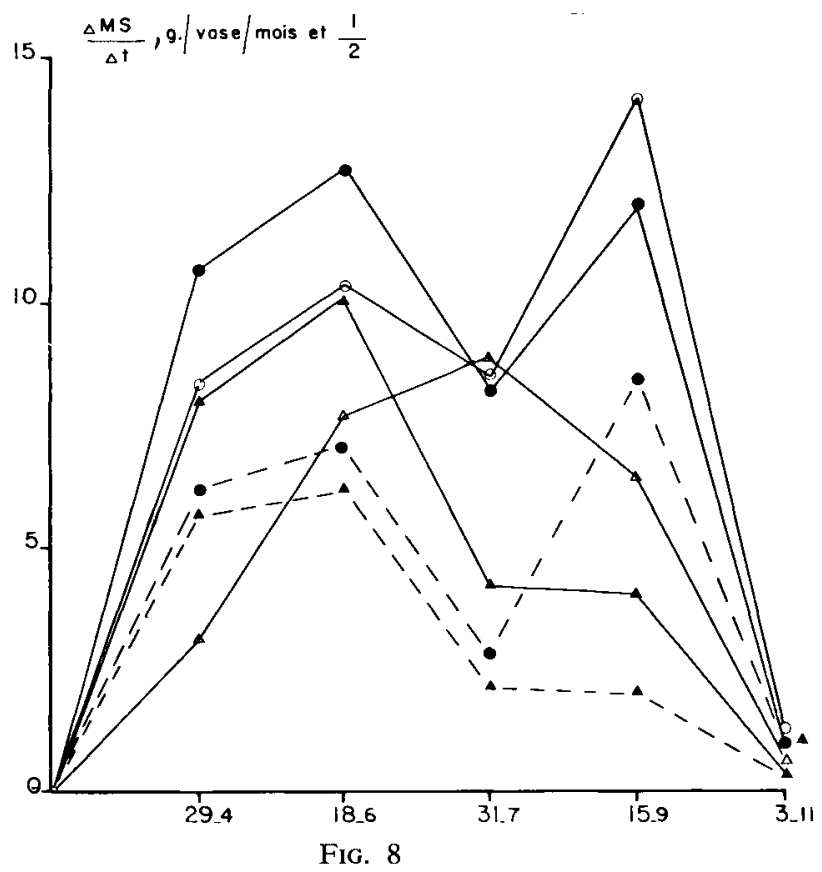

Interaction éclairement-déficit hydrique sur la croissance de la molinie en vase de végétation. Régime moyen et sec. Evolution des productions «mensuelles》 (5 coupes).

Effect of interaction between radiation and water deficit upon the Molinia growth in pots. Dry and average water supplies. Evolution of monthly productions.

E T M

$P_{\text {lux }}=1$ extérieur

$P_{\text {lux }}=0,60$ (serre)

$P_{\text {lux }}=0,30$ (extér.)

$P_{\text {lux }}-0,26$ (serre)
$\mathrm{E} \mathrm{T} \mathrm{M} / 2$

- l'effet sécheresse se fait sentir tout au long de lannée, et tout particulièrement en période estivale comme le révèle la superposition des courbes ETM et. ETM

2

- l'interaction ombrage-sécheresse est différente selon la saison : au printemps. ETM

un ombrage modéré $\quad P_{0,26}$ pour $\frac{}{2}$ intervient peu, par contre à l'automne

il entraîne une très forte chute de la production. Il se peut donc que, dans ce dernier cas, la plante ait besoin d'une énergie assez élevée pour récupérer l'effet de stress estival.

\subsection{Amendement et fertilisation de la molinie}

Afin d'explorer les possibilités d'amélioration fourragère du sous-bois on a procédé en deux étapes : 
- une étape préalable ayant conduit à éliminer provisoirement la possibilité d'avoir recours sur ce type d'écosystème à des espèces cultivées, même en ayant recours à l'amendement calcique (cf. \$ 2.2);

- une étape finale destinée à déterminer les exigences minćrales de la molinie sur le sol de lande humide, et par-là même à déboucher sur une formule économique.

Les interactions entre éléments minéraux y ont été envisagées de la façon la plus large possible.

\subsection{Réaction au chaulage des espèces fourragères cultivées en sol forestier des Landes}

A l'origine, le dispositif expérimental mis en place à Pissos en vue d'étudier les conditions de l'implantation d'un élevage sous forêt reposait sur une production d'herbe à partir de plantes prairiales introduites moyennant un apport modeste de fertilisants et d'amendements calciques. L'extrême acidité du sol constituant l'un des principaux facteurs de blocage au développement des végétaux cultivés, on a, lors de la première phase de l'étude, expérimenté en serre le comportement des plantes fourragères mises en œuvre dans le dispositif en présence de doses croissantes de dolomic (hiver 1975-1976).

La dose de chaux qui a permis de parvenir au rendement optimal s'est située aux environs de $500 \mathrm{~kg} / \mathrm{ha}$ de $\mathrm{CaO}$, dose qui a permis un gain de production de 50 p. 100 en moyenne; les légumineuses testées (trèfle blanc et lotier des marais) réagissent le mieux $(+70$ p. 100) et les graminées (4 fétuques élevées et 3 dactyles) un peu moins $(+30$ p. 100). Cet essai a également permis de mettre en évidence des différences très nettes de composition minérale entre Fétuque et Dactyle, ces derniers apparaissant comme de meilleurs accumulateurs de minéraux (notamment en éléments mineurs) et azote.

Le maintien de ces sols à un niveau calcique satisfaisant apparaît donc comme primordial pour valoriser les fertilisations minérales, même modestes, utilisées. Le lessivage de la chaux dans des formations comparables a pu être évalué à $300 \mathrm{~kg} / \mathrm{ha} / \mathrm{an}$ dans le cas de cultures intensives irriguées; compte tenu du caractère extensif du système étudié ici, et de la couverture permanente du sol, la dose de $250 \mathrm{~kg} / \mathrm{ha} / \mathrm{an}$ de $\mathrm{CaO}$ apparaît comme un minimum à épandre pour sauvegarder la végétation introduite.

Le suivi du dispositif expérimental ayant fait apparaître l'impossibilité de maintenir les graminées fourragères semées dans les conditions de système extensif, on a décidé, lors d'une deuxième phase, de mesurer l'effet des matières fertilisantes sur la végétation spontanée utilisable (molinie). Les expériences ont été réalisées en premier lieu à partir de molinie reproduite en serre par voie de semis.

\subsection{Réaction à la chaux et à l'azote de la molinie reproduite par voie de semis}

La première coupe, réalisée 3 mois environ après la levée des plantes, fait apparaître un effet significatif mais très modeste de la plus forte dose d'azote expérimentée (équivalent de $150 \mathrm{~g} / \mathrm{ha}$ ); par contre, la chaux appliquée à 1000 unités de $\mathrm{CaO} / \mathrm{ha}$ déprime les rendements.

La seule exploitation des résultats de cette expérience aurait donc conduit à déconseiller des apports de chaux sur molinie et à poser la question de l'opportunité 
économique de l'utilisation d'engrais azotés, compte tenu de la très faible efficacité de ces derniers. En fait, la $2^{\prime \prime}$ coupe réalisée 4 mois et demi après la première remet en question cette conclusion provisoire, l'effet de l'azote étant pratiquement multiplié par 6 à 9. Par ailleurs, si la chaux appliquée seule continue à se montrer sans effet, l'application conjointe de l'amendement et de l'engrais azoté provoque à la $2^{\text {e coupe }}$ un accroissement spectaculaire du rendement.

Cette première expérience avec molinie met donc en évidence l'effet très retardé de l'azote des engrais : l'enrichissement considérable de la plante en cet élément à la première coupe malgré l'absence de réaction permet-il de supposer que l'azote doit préalablement subir au sein de la graminée des transformations qui le rendent apte à exercer son action sur la croissance ? Cette expérience fait aussi ressortir l'inopportunité d'un apport d'amendement calcaire sur molinie, non associé à de l'azote.

\subsection{Réaction au phosphore et au potassium de la molinie reproduite par voie de semis}

Le recoupement de l'essai «azote et chaux» par des apports de potassium et de phosphore, séparés ou conjoints, à un sol pratiquement dépourvu de ces éléments entraîne des augmentations immédiates mais modérées de rendement, avec un effet de synergie extrêmement net.

\subsection{Réaction à lazote, au phosphore et au potassium de la molinie reproduite par «éclats». Comparaison avec la fétuque (fig. 9)}

La technique d'installation de la molinie par éclats permet un démarrage beaucoup plus rapide de la plante et, de ce fait, accélère la collecte d'informations. Cette expérience confirme leffet retardé de l'azote, qui ne se manifeste que 90 jours après mise en place des éclats. Elle confirme aussi la synergie NPK, dont l'intensité est telle qu'il apparaît comme peu réaliste d'escompter un effet de l'azote en sol pauvre en potasse et phosphore si l'on n’effectue pas un apport, même modeste, de ces deux éléments.

Pour un niveau de fertilisation équivalent à 150 unités/ha de $\mathrm{N}$ et 100 de $\mathrm{P}$ et $\mathrm{K}$, la production cumulée de matière sèche de la molinie ( 5 coupes dans l'année) représente 75 p. 100 de celle de la Fétuque, et cela pour des densités de peuplement fort différentes (semi serré de Fétuque et implantation lâche des éclats de molinie).

L'extrapolation à l'hectare de la production de matière sèche de molinie conduit à une estimation de $2,68 \mathrm{t} /$ ha pour le témoin non fertilisé, soit une valeur d'un ordre de grandeur déjà rencontré sur le terrain (cf. $\$ 2.31$ ); l'apport d'une fertilisation de 100 unités de phosphore et de potasse et de 75 unités d'azote double pratiquement cette production à l'échelle de l'année.

Le passage de l'équivalent $75 \mathrm{u} /$ ha de $\mathrm{N}$ à $150 \mathrm{u} /$ ha ne provoque qu'une augmentation de 13 p. 100 de la production. Loptimum économique de la fertilisation azotée (impérativement associée à $\mathbf{P}$ et $\mathrm{K}$ ) pourrait donc se situer, pour la molinie, aux environs de $75-100 \mathrm{u} /$ ha de $\mathrm{N}$.

Sur le plan qualitatif, on observe que lapport dune fertilisation incluant du potassium diminue sensiblement la teneur en matière sèche de la molinie. Par ailleurs, 
à dose égale de fertilisants appliqués, la molinie se montre meilleur accumulateur d'azote, de potassium, de cuivre et de manganèse que la Fétuque, alors que l'inverse est observé pour le magnésium et surtout le calcium. Parallèlement à cet essai, et dans les mêmes conditions d'installation, on constate à nouveau le caractère nettement calcifuge de la molinie.

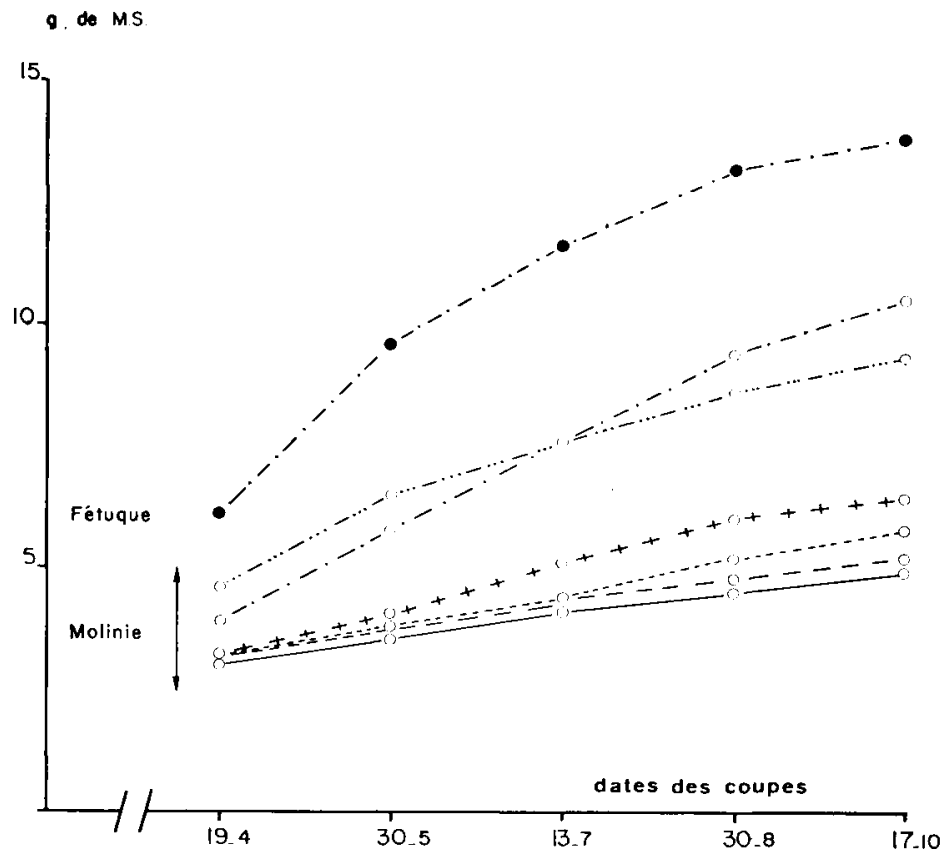

FIG. 9

Ferrilisation minérale de la molinie (éclats) avec un témoin fétuque (semis).

Essais 1979 en vase de végétation sur sol de lande. Production cumulée de matière sèche.

Mineral fertilization of Molinia compared with a Fescue control.

Grow'th curves of dry weight.

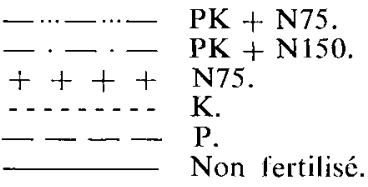

2.45. Conclusions provisoires

Dans la mesure où la valorisation par l'élevage du sous-bois forestier de la pinède landaise doit obligatoirement passer par l'utilisation des graminées spontanées, et principalement de la molinie, on peut raisonnablement proposer une fertilisation 
modérée équivalent à un apport initial de 75 à 100 unités/ha d'azote, associé à 100 unités de potassium et d'acide phosphorique. Il apparaît ainsi possible de doubler pratiquement la production de la graminée et d’améliorer sa qualité (diminution de la teneur en matière sèche, accroissement sensible de la richesse en azote et en éléments mineurs).

L'apport d'amendement calcaire apparaît sans intérêt, surtout en présence d'un système racinaire puissant ou en développement rapide (touffes ou éclats) permettant une alimentation azotée intense.

Cette première approche du fonctionnement du peuplement molinie demanderait à être complétée par l'acquisition d'informations concernant notamment l'arrière-effet des fumures après le repos hivernal et l'influence du rythme d'exploitation de la graminée sur sa productivité ultérieure.

\subsection{Effet du pâturage sur le végétal}

L'effet du pâturage sur chacune des deux strates a été étudié dans les sites suivants :

- Pissos pour la pelouse (Bergeret H., 1980) et pour le peuplement d'arbres (résultats en cours d'obtention);

- Cestas pour le peuplement d’arbres.

\subsection{Effet sur la pelouse}

Les résultats sont dordre qualitatif et quantitatif. Ils ont trait au site A de lande humide de Pissos.

Pour les 4 traitements pâturés, les mesures sont effectueés dans des enclos ayant soustrait la végétation à laction de l'animal (cf. Bergeret H., 1980). Les données ont donc trait à des $\frac{\Lambda \mathrm{MS}}{\Delta \mathrm{t}}$ successifs obtenus sur plusieurs parcelles pendant deux ans.

Sur le plan qualitatif : afin d’interpréter globalement toutes les situations rencontrées on a procédé à la définition d'une séquence de pâturage (DE MONTARD F.X., communication personnelle), c'est-à-dire d'un transect ayant son origine dans un site non pâturé et s'en éloignant jusqu'à un point d'affouragement; l'annexe 2 donne le détail des relevés floristiques effectués aux 5 points de cette séquence ct la figure 10 en fait la synthèse : les 2 pôles de la séquence sont à tendance "monospécifique 》molinie ou espèces introduites - à l'inverse des parties médianes.

$$
\text { Sur le plan quantitatif : le modèle d'explication des } \frac{\Delta \mathrm{MS}}{\Delta \mathrm{t}} \text { par les facteurs }
$$

climatiques (cf. équations VI et VII du tab. 3 et fig. 11) a été complété par la mise en œuvre d'un paramètre rendant compte globalement des effets du pâturage sur la pelouse de molinie - $\mathrm{J} / \mathrm{T}$ et $(\mathrm{J} / \mathrm{T})^{2}-$ combinant le nombre d'animaux - Jours $\mathrm{J}$ de pâturage et le temps écoulé $T$ depuis la dernière pâture. On remarque ainsi que 
l'apport de ce paramètre, selon un ajustement parabolique, est globalement significatif — alors qu'il ne l'est pas selon un ajustement linéaire - ; ce fait exprime, par exemple, que la production d'une parcelle fortement pâturée évolue, après sa mise en défens, d'abord en subissant un effet dépressif ( $\mathrm{J} / \mathrm{T}$ forts) puis passe par un maximum $(\mathrm{J} / \mathrm{T}=17)$, enfin diminue progressivement $(\mathrm{J} / \mathrm{T}$ de plus en plus faibles) jusqu'à une production équivalente à celle d'une parcelle non pâturée. Mais nous ne savons pas si une telle parcelle, pâturée plus longtemps, aurait manifesté les mêmes facultés de récupération : se trouvait-elle, avant sa mise en défens, dans un état d'équilibre stable - entre biomasse sur pied, croissance et consommation - ou bien était-elle proche d'une évolution vers une altération de ses capacités productives? Noy-MelER (1975) a développé tous les aspects théoriques de ce type dans une application du modèle «proie-prédateur» aux prairies pâturées.

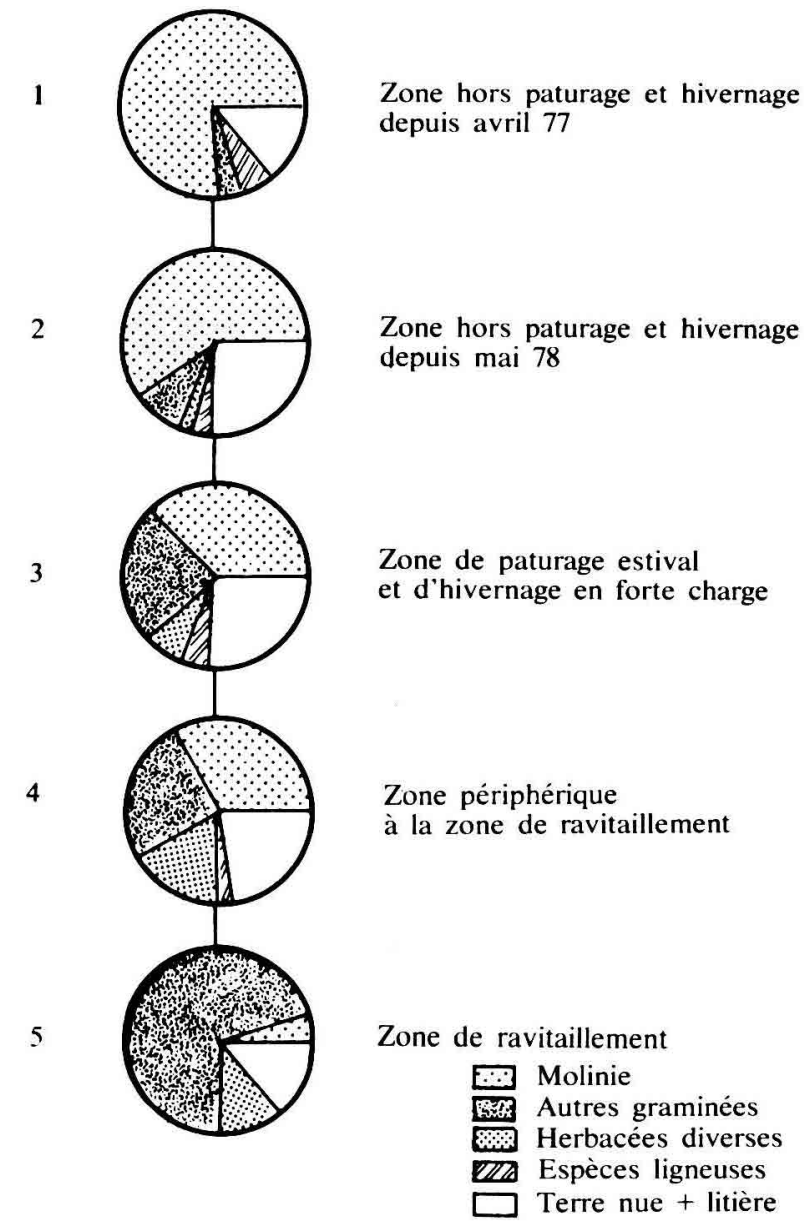

FIG. 10

Diagrammes floristiques d'une séquence de pâturage. Site A de Pissos. Floristic diagramms of a grazing sequence. 


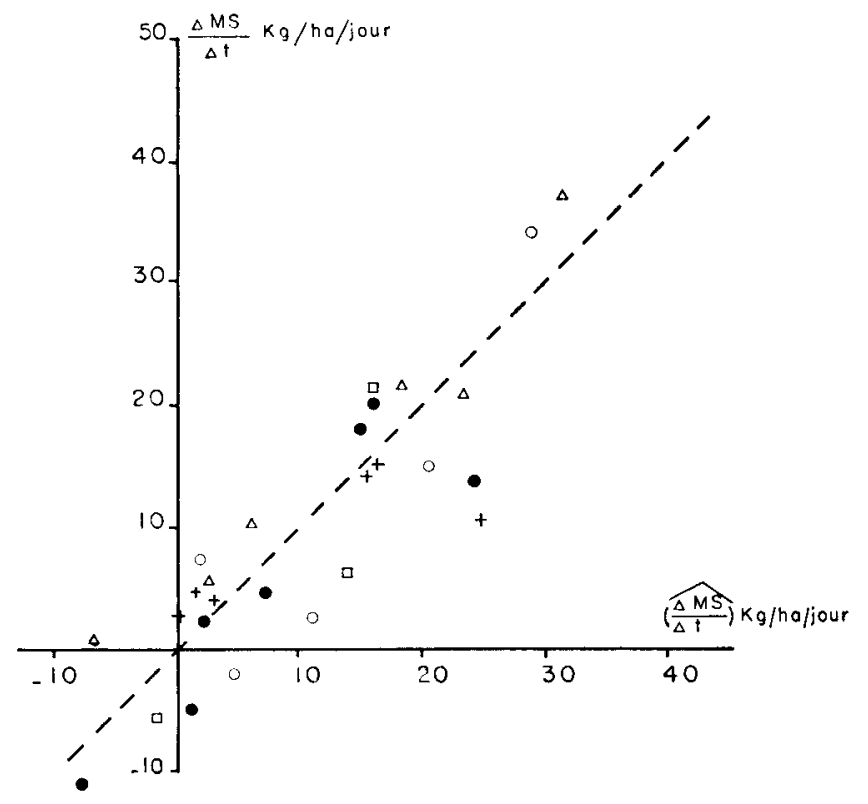

FIG. 11

Production de la pelouse en fonction de l'ETP, du déficit en eau et du pâturage. Site $A$ de Pissos.

Confrontation des valeurs mesurées aux valeurs calculées par le modèle: équation $I X$ du tableau 3 :

$\Delta \widehat{\mathrm{MS}}$

$\Delta \mathrm{t}$

$=-26,3+38,7 \cdot \frac{\Delta \mathrm{ETP}}{\Delta \mathrm{t}}-105 \cdot \frac{\Delta \mathrm{ETP}-\Delta \mathrm{ETRO}}{\Delta \mathrm{ETP}}+1,19 \cdot \frac{\mathrm{J}}{\mathrm{T}}-0,035 \quad\left(\frac{\mathrm{J}}{\mathrm{T}}\right)^{2}$

Meadow yield as a function of ETP and water deficit.

Measured observed values versus estimated ones.

The latter were estimated by a regression model.

- Non pâturé, P11.

O Hivernage faible charge, $\mathrm{PH} 2$.

+ Pâturage estival, P9.

$\triangle$ Hivernage forte charge, PH1.

$\square \quad$ Hivernage très forte charge, $\mathrm{PH}^{\prime} 1$.

Signalons que Warren (1979) constate une absence d'effet significatif du pâturage sur la pousse de l'herbe, alors que les traitements mis en ouvre par cet auteur font varier les prélèvements bruts par l'animal de 30 à 60 p. 100, donc de 0 p. 100 (témoin) à 60 p. 100 (fortement pâturé) ; en revanche la composition botanique est significativement modifiée. 


\subsection{Effet sur le peuplement d'arbres}

Le peuplement de pins maritimes est issu d'une régénération naturelle sur incendie de 1949. Des interbandes y ont été ouvertes ultérieurement. Le site est une lande mésophile humide.

Le dispositif comprend 5 blocs et 4 traitements :

- témoin, sans aucune fertilisation ni façons culturales, T.A.;

- traitement ayant reçu une fertilisation de fonds enfouie par des façons superficielles, T.F.;

- traitement ayant reçu la même fertilisation de fonds et les mêmes fertilisations d'entretien que les prairies artificielles, T.F.E.;

- prairies artificielles de Dactyle ou de Fétuque avec fertilisations de fonds et d'entretien enfouie par des façons superficielles, P.; seul ce traitement est pâturé.

\section{Tableau 4}

Effet de l'animal sur le peuplement d'arbres.

Accroissements en surface terrière durant 2 périodes de 3 ans.

Résultats d'analyses de covariance (covariable = surface terrière ST génératrice).

Effect of the animal upon the trees stand. Basal area increments during 2 periods. Covariance analysis with basal area as covariable.

1) Période 73-76 :

$\begin{array}{lrcccc}\text { a) Moyennes ajustées : traitements } & : & \mathrm{P} & \mathrm{TF} & \text { TFE } & \text { TA } \\ \Delta \mathrm{ST}, \mathrm{m}^{2} / \mathrm{ha} & : & 6,77 & \overline{\mathbf{5}}, 34 & 5,69 & \mathbf{4 , 3 5}\end{array}$

b) Différences entre traitements

$\begin{array}{llll} & \text { TF } & \text { TFE } & \text { P } \\ \text { TA } & 1,00^{* * *)} & \left.1,34^{* * * *}\right) & 2,42^{* * * *} \\ \text { TF } & & 0,35 & 1,43^{* *(*)} \\ \text { TFE } & & & 1,08^{* *(*)}=\text { P - TFE }\end{array}$

2) Période 76-79:

a) Moyennes ajustées : traitements : $\quad P \quad$ TF $\quad$ TFE $\quad$ TA $\begin{array}{llllll}\Delta \mathrm{ST}, \mathrm{m}^{2} / \mathrm{ha} & : & 4, \mathrm{j} 8 & 4.08 & 4,3 \mathrm{j} & 4,13\end{array}$

b) Différences entre traitements :

$\begin{array}{llll}\text { TA } & 0,04 & 0,26 & 0,50 *(*) \\ \text { TF } & & 0.22 & 0,46^{* *(*)} \\ \text { TFE } & & & 0,24\end{array}$

:**: : Probl. $=1$ p. 1000.

$* *(*): 1$ p. $1000<$ Probl. $<1$ p. 100.

$*(*): 1$ p. $100<$ Probl. $<5$ p. 100. 
Le système de pâturage adopté au début était relativement intensif -1050 jours ovin/ha/an. Or il s'est avéré que la prairie artificielle régressait rapidement au profit de la flore naturelle. Dans une deuxième période le système d'exploitation est alors devenu extensif - réduction de la fertilisation et du nombre de jours de pâturage soit 450 jours/an. Les chablis de 1976 ont compliqué l'exploitation des résultats : les accroissements en surface terrière, ou en volume, des placeaux unitaires sont analysés par l’analyse de covariance - la covariable est la surface terrière génératrice - ce qui permet également de tenir compte des différences de densités initiales des placeaux unitaires (tabl. 4).

Sur le plan strictement sylvicole -- comparaison des traitements T.A. d'une part, T.F. et T.F.E. d'autre part - on a affaire à un effet de fertilisation différée conforme aux résultats déjà acquis (LEFrou G., 1980). La fertilisation appliquée dans ce système d'exploitation de la prairie bénéficie donc également au peuplement d'arbres.

Sur le plan de la relation animal-arbre on constate que laccroissement en surface terrière du traitement $P$ est supéricur à celui du traitement T.F.E. : cette supériorité est de 19 p. 100 ( $^{*}$ ) pendant la période $1974-1976$ et de 5 p. 100 (N.S.) pendant la période 1977-1979.

On constate donc un effet positif global du pâturage sur la croissacne du peuplement de pins sans qu'il soit possible de faire les parts respectives de l'effet prairie et de l'effet propre à l'animal. On remarque par ailleurs que cet effet global est proportionné à l'intensité du système d’exploitation (comparaison des périodes).

\section{Discussion et conclusion}

Ce travail constitue une première approche de l'écosystème landais vu à travers la problématique de l'élevage en forêt.

Si bien des connaissances scientifiques restent à acquérir. le déroulement de cette recherche permet néanmoins, au vu des résultats acquis, de formuler certaines considérations provisoires d'ordre technicoéconomique :

- la productivité de l'écosystème forestier perdrait beaucoup à une orientation de la gestion sylvicole faite dans le sens dune augmentation de la production de la pelouse; l'augmentation de production de celle-ci en fonction de la diminution du couvert, bien qu'elle soit relativement bonne, ne le justifie pas pour l'instant; sur ce milieu qui se révèle pauvre vis-à-vis du sous-bois (contrairement à ce qui se passe pour le pin maritime), on constate, d'une part une productivité modeste de la flore spontanée, d'autre part un mauvais pouvoir compétitif des flores introduites vis-à-vis de cette dernière; on ne pourra revenir sur cette conclusion quà la condition de trouver à introduire des espèces ou des variétés (existant déjà ou non) productives et adaptées tout à la fois, à l'hydromorphie printanière, au manque relatif de lumière et à la sécheresse d'été;

- la molinie réagit bien, et de façon synergique, à lapport de plusieurs éléments minéraux; on peut donc en principe conseiller une formule de fertilisation modérée ; il n'en reste pas moins vrai que si cet investissement est faible dans laabsolu, il risque d'être dans notre contexte à la limite de la rentabilité ; cette réserve étant faite, on ne 
devra pas oublier que nous ne savons pas encore quel est l'arrière-effet d'une telle fumure ; dans l'hypothèse favorable, et dans le cas où le pin maritime "réclamerait» un renouvellement des doses (ce que l'on ne sait pas encore), on pourrait conclure à une rentabilité globale de l'investissement fumure; par la suite même, l'animal pourrait maintenir la productivité de la pelouse par le jeu des turnovers minéraux, à condition que la charge requise à cet effet ne dépasse pas le seuil, que nous avons déjà perçu, au-delà duquel l'influence de l'animal devient négative ; l'éventualité d'une fertilisation minérale pourrait être envisagée chaque fois que la surface disponible du sous-bois serait un facteur limitant;

- les effets du pâturage sur la croissance du peuplement de pins n'ont pu encore être complètement analysés; on remarque déjà qu'à charge moyenne ou faible, ils ne sont pas négatifs ; un effet propre positif (distingué de l'effet prairie) serait extrêmement intéressant puisqu'il améliorerait, dans la même optique que ci-dessus, la rentabilité globale du système.

On est donc amené à prendre deux attitudes : d'une part à court terme il n'y a pas d'inconvénient majeur à la superposition d'un élevage extensif à la sylviculture landaise, d'autre part à moyen et à long terme, il n'est pas interdit de penser que les résultats de la recherche conduisent à un système plus intégré.

Sur le plan méthodologique on peut formuler les remarques suivantes:

- caractériser complètement la productivité d'une plante fourragère implique bien évidemment de connaître l'impact d'un nombre suffisant de rythmes d'exploitation; nous nous sommes limités jusqu'à présent pour la végétation naturelle en place à quelques modèles simples ( 1 ou 2 coupes) ;

- le modèle d'explication bioclimatologique de la croissance de la molinie est perfectible de la façon suivante : meilleure caractérisation de la réserve utile en eau, mesure directe de l'ETP (rayonnement net) et peut-être prise en compte du recouvrement de l'herbe ;

- nous avons tenté une approche de l'action de l'animal sur l'herbe en mesurant ses «arrières effets » après mise en défens; on a pu ainsi modéliser cet aspect de son action en termes d'équilibre écologique; les travaux se poursuivent actuellement en situation de pâturage actuel, et non plus seulement récent; il resterait, une fois nos écosystèmes bien caractérisés, à apprécier la consommation de l'animal; cette exigence est d'autant plus impérative que la pression du pâturage est grande. Or nous pouvons être amenés à travailler sur un système plus intensif, au moins pendant certaines périodes de l'année.

Reçu pour publication en avril 1982.

\section{Remerciements}

Nous remercions tout particulièrement :

F.X. DE Montard qui nous a conseillé tout au long de ces travaux; M. Ducrey qui a effectué pour nous le dépouillement des photographies hémisphériques ;

La Compagnie d'Aménagement Rurale d'Aquitaine (C.A.R.A.) chez laquelle une partie des expérimentations a pu être installée ;

Nos techniciens G. LeFrou et A. Sartolou;

$\mathrm{M}^{\mathrm{me}}$ Gulliem et $\mathrm{J}$. Darnaudery pour la dactylographie et les dessins;

Tous ceux qui ont collaboré avec nous. 


\section{Summary \\ Stock farming in the forests of the «Landes de Gascogne». \\ I. The vegetation system}

Two objectives are followed in this study. On one hand we try to determine grass yield of underwood in humid moor. On the other hand we try to analyse the functioning of this ecosystem which is made up of two vegetal stories : pine stand and underwood.

Following results are pointed out :

a) cultivated fodder plants are not competitive against natural plants mainly Molinia coerulea M. :

b) the dry weight productivity of Molinia in low, between 0.7 and 1.8 tonne per ha and per year. Molinia is available to the cattle only during 5 to 6 months. The productivity varies as a function of the forest stand density. It is dependent upon the availability of mineral elements by fertilization, mainly $\mathrm{N}$ which interacts with $\mathrm{P}$ and $\mathrm{K}$. We plan to use this underwood extensively ;

c) data of periodic grass production can be fitted with sufficient accuracy by a multiple regression of two climatic parameters, ETP and water deficit, these parameters being modified by the forest stand;

d) the influence of the animal on grass is interpreted as a "grazing sequence»;

e) overall the forest stand takes benefits of the harwest of the artificial cultivated grass by cattle.

\section{Références bibliographiques}

Allart, 1979. "Contribution à l'étude de la valeur nutritive des plantes fourragères du sous-bois landais ». Rapport de stage E.N.I.T.A. Bordeaux.

Aussenac G., 1980. Interception des précipitations par le couvert forestier. Ann. Sci. for., 25 (3), 135-166.

Bergeret H., 1977 Influence du milieu et du pâturage sur la production de molinie en forêt landaise. D.E.A. Université Bordeaux III, $103 \mathrm{p}$.

BERGERET H., 1980. Evolution floristique et productivité de Landes à molinie (Molinia coerulea M.) en forêt landaise. Influence de traitements agronomique et sylvicole. Doctorat $3^{\circ}$ cycle. Université Bordeaux III, $154 \mathrm{p}$.

BoucheT P., 1964. Evapotranspiration potenticlle et production agricole. L'eau et la production végétale. I.N.R.A.

Brochet P., Gerbier N., 1972. Une méthode pratique de calcul de l'évapotranspiration potentielle. Ann. agron., 23 (1). 31-49.

Cabanettes A., Rapp, 1978. Biomasse, minéralomasse et productivité d'un écosystème méditerranéen. I. - Biomasse. Ecol. Plant., 13 (3), 271-286.

Commeres A., 1980. Valeur nutritive et alimentaire des plantes fourragères en sous-bois landais. Mémoire-thèse E.N.I.T.A. Bordeaux.

Comps B., Geipe J., Saint-Didier J., 1979. Nouvelle typologie des Landes de Gascogne : Modèle d'étude sur la commune de Pissos (Landes). Bull. Ecol., 10 (1), 43-54.

Decourt N., Lemoine B., 1969. Tables de production provisoires pour le pin maritime dans le Sud-Ouest de la France. Ann. Sci. for., 26 (1), 3-44.

De Montard F., 1981. L'action des facteurs climatiques sur la croissance de l'herbe. Exemple d'une prairic à Agrostis tenuis et à Poa pratensis des monts d'Auvergne. Fourrages, 85, mars. 
Ducrey M., 1975. Utilisation des photographies hémisphériques pour le calcul de la perméabilité des couverts forestiers au rayonnement solaire. I. - Analyse théorique de l'interception. Ann. Sci. for, 32 (2), 73-92.

Farnsworth M.C., 1979. Wind velocity reduction in a forest farm stand. Farm Forestry, $21(2), 35-40$.

Galley C., 1977. Contribution à la connaissance de la valeur nutritive des fourrages produits sous couvert forestier. Mémoire-thèse E.N.I.T.A. Bordeaux, 76 p.

Halles L.K., Suman R.F., 1954. Improved forage under southern pines. J. Forest., 52, 11.

Jeannin B., Garel J.P., Louyot J., de Montard F., Petit M., 1979. Production et utilisation rationnelle des pâturages d'altitude dans les montagnes humides du Massif Central. In : Utilisation par les ruminants des pâturages d'altitude et des parcours méditerranéens, Public. I.N.R.A.

Lefrou G., 1980. Résultats, après 10 ans d'expérimentation, de deux essais de fertilisation de regonflage sur pin maritime dépérissant dans les Landes de Gascogne. Rev. For. fr., 31 (2), 127-134.

Lemoine B., Sartolou A., 1976. Les éclaircies dans les peuplements de pin maritime d'âge moyen. Résultats et interprétation d'une expérience. Rev. For. fr., 26 (6), 447-457.

Lemorne B., 1980. Densité de peuplement, concurrence et coopération chez le pin maritime. II. - Résultats à 5 et 10 ans d'une plantation à espacement variable. Ann. Sci. for., 37 (3), 217-237.

Leredde C., 1947. Notice sur la lande et le sous-bois des Landes de Gascogne. Congrès de l'A.F.A.S. Ed. Douladoure, Toulouse, 7 p.

Miqueux M., 1979. Production d'été de variétés de fétuque élevée en fonction des facteurs climatiques. Ann. Anél. Plant., 29 (1), 97-105.

Noy-Meir I., 1975. Stability of grazing systems : an application of predator - prey graphs. J. Ecol., 63 (2).

RANGER J., 1978. Recherches sur les biomasses comparées de deux plantations de pin Laricio de Corse avec ou sans fertilisation. Ann. Sci. for., 35 (2), 93-115.

Robelin M., Collier D., 1958. Evapotranspiration et rendements culturaux. C.R. Acad. Sci., 254, 718-720.

Robelin M., 1969. L'alimentation en eau des plantes fourragères. Fourrages, 38, 30-40.

Turc L., 1961. Evaluation des besoins en eau d'irrigation, évapotranspiration potentielle, formule simplifiće et mise à jour. Ann. agron., 12 (1), 13-49.

Warren P., Clary, 1979. Grazing and Overstory effects on rationally burned slash pine plantation ranges, J. Range Manage, 32 (4), 264-266. 
Tableau floristique des espèces présentes dans 6 placeaux « mis en défens» à Pissos : Présence. Abondance (in BERGERET, 1980). (Contribution spécifique au recouvrement).

\begin{tabular}{|c|c|c|c|c|c|c|c|c|c|c|}
\hline \multirow{2}{*}{$\begin{array}{c}\text { Parcelles } \\
\text { Dates }\end{array}$} & \multicolumn{5}{|c|}{$P_{11}$} & \multicolumn{4}{|c|}{$\mathrm{PH}_{2}$} & \multirow[b]{2}{*}{$\sum^{3} \frac{N}{\sigma}$} \\
\hline & $\sum_{i}^{\pi} \frac{1}{2}$ & $\stackrel{\vec{\Xi}}{\Xi} \stackrel{\sim}{\Xi}$ & $\sum \frac{\infty}{i}$ & 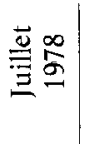 & 总 & 芯卡 & 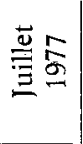 & 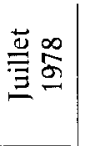 & 冚恖 & \\
\hline Litière terre nue $\ldots \ldots \ldots \ldots \ldots$ & 23 & 21 & 49 & 9 & 24 & 40 & 23 & 7 & 26 & 60 \\
\hline $\begin{array}{l}\text { Strate arborescente } \\
\text { Pinus maritima } \ldots \ldots \ldots \ldots \ldots\end{array}$ & + & + & + & + & + & + & + & + & + & + \\
\hline $\begin{array}{l}\text { Strate arbustive } \\
\text { Ulex nanus ............. } \\
\text { Erica scoparia } \ldots \ldots \ldots \ldots \ldots \ldots \\
\text { Rhanmus frangula } \ldots \ldots \ldots \ldots \ldots \\
\text { Pteris aquilina } \ldots \ldots \ldots \ldots \ldots \ldots \\
\text { Ulex europaeus } \ldots \ldots \ldots \ldots \ldots \ldots\end{array}$ & $\begin{array}{c}0,4 \\
+ \\
+\end{array}$ & $\begin{array}{c}5 \\
5,5 \\
+\end{array}$ & $\begin{array}{l}+ \\
+ \\
+\end{array}$ & $\begin{array}{l}+ \\
+ \\
+\end{array}$ & $\begin{array}{c}+ \\
1 \\
+\end{array}$ & $\begin{array}{c}3,2 \\
7,8 \\
+\end{array}$ & $\begin{array}{c}1,6 \\
5,0 \\
+\end{array}$ & $\begin{array}{c}5 \\
8 \\
+\end{array}$ & $\begin{array}{r}4 \\
2 \\
+\end{array}$ & $\begin{array}{c}4,4 \\
1,6 \\
+ \\
1,6\end{array}$ \\
\hline 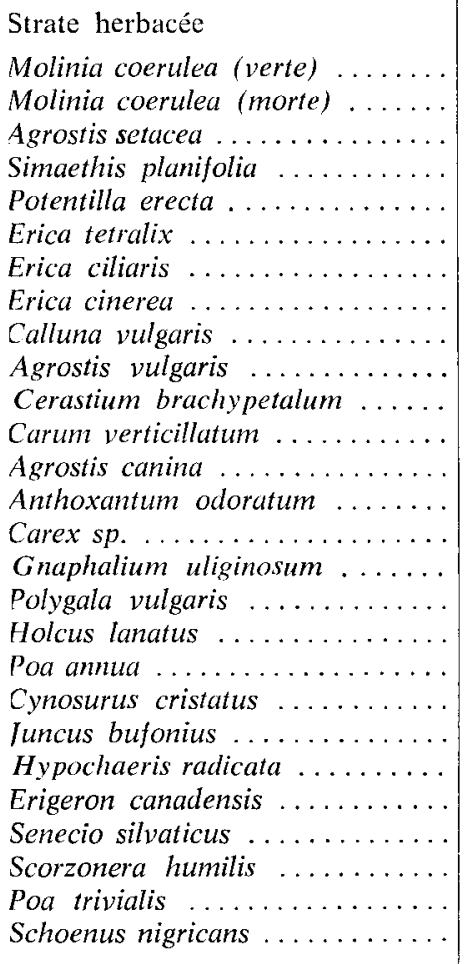 & $\begin{array}{l}30 \\
46 \\
0,4 \\
\bullet \\
-\end{array}$ & $\begin{array}{l}52 \\
16 \\
+ \\
+ \\
-\end{array}$ & $\begin{array}{r}28 \\
13 \\
3 \\
6 \\
1\end{array}$ & $\begin{array}{r}39 \\
49 \\
3 \\
+ \\
-\end{array}$ & $\begin{array}{l}44 \\
31 \\
+ \\
+ \\
+\end{array}$ & $\begin{array}{r}36 \\
6 \\
0,1 \\
4,6 \\
. \\
. \\
. \\
.\end{array}$ & $\begin{array}{c}65 \\
\bullet \\
3,3 \\
1,6 \\
\bullet \\
\bullet \\
\bullet\end{array}$ & $\begin{array}{c}39 \\
40 \\
+ \\
0,3 \\
0,7 \\
+ \\
+ \\
+\end{array}$ & $\begin{array}{c}15 \\
50 \\
3 \\
+ \\
+ \\
\cdot \\
\cdot \\
1\end{array}$ & $\begin{array}{c}21 \\
6 \\
1 \\
1,6 \\
\bullet \\
\bullet \\
\bullet \\
\bullet \\
\\
1,6 \\
+\end{array}$ \\
\hline
\end{tabular}

$\mathbf{P}_{11}$ : Témoin non pâturé.

$\mathrm{PH}_{2}$ : Hivernage faible charge.

$P_{9}$ : Pâturage estival.

$\mathrm{PH}_{1}$ : Hivernage forte charge.

$\mathrm{PH}_{1}^{\prime}$ : Hivernage très forte charge.

$P_{10}$ : Pâturé et fertilisé. 
Floristical table of species in 6 plots against cattle protected in Pissos : Presence. Abundance (recoveral).

\begin{tabular}{|c|c|c|c|c|c|c|c|c|c|c|c|c|c|c|}
\hline \multicolumn{3}{|c|}{$P_{9}$} & \multicolumn{3}{|c|}{$\mathrm{PH}_{1}$} & \multicolumn{3}{|c|}{$\mathrm{PH}_{1}^{\prime}$} & \multicolumn{6}{|c|}{$P_{10}$} \\
\hline$\stackrel{\overrightarrow{0}}{\stackrel{\Xi}{\Xi}}$ & $\stackrel{\vec{e}}{=} \stackrel{\infty}{\Xi}$ & 营各 & 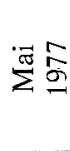 & 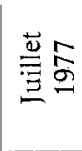 & $\sum^{\pi} \stackrel{\infty}{\vdots}$ & 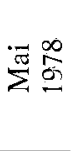 & 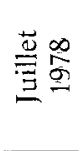 & 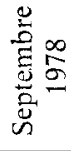 & $\sum^{-\pi} \frac{N}{\sigma}$ & 弮鸽 & $\sum^{\vec{\pi}} \frac{\infty}{2}$ & $\stackrel{\bar{\Xi}}{\stackrel{0}{\Xi}}$ & 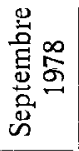 & 范昜 \\
\hline 18 & 8 & 35 & 44 & 16 & 14 & 52 & 27 & 38 & 31 & 7 & 8 & 5 & 8 & 20 \\
\hline+ & + & + & + & + & + & + & + & + & + & + & + & + & + & + \\
\hline 12 & 5,4 & 4 & 4,6 & 8,3 & 3 & 4 & 1 & 1 & 9 & 12 & 7 & 10 & 12 & 5 \\
\hline 3,3 & 3,5 & + & 0,9 & 6,1 & + & 1 & + & 3 & 2 & 7 & 5 & 1 & + & + \\
\hline+ & + & + & + & + & + & + & + & $t$ & + & 1 & + & + & 1 & + \\
\hline 2,2 & + & + & + & + & + & + & + & + & + & + & 5 & 2 & + & 2 \\
\hline 57 & 39 & 22 & 44 & 68 & 46 & 38 & 61 & 51 & 44 & 55 & 39 & 46 & 33 & 27 \\
\hline+ & 34 & 32 & 4 & . & 30 & & & & 7 & • & 25 & 23 & 36 & 40 \\
\hline+ & + & + & 2,7 & 1,6 & 1 & 1 & 1 & + & + & + & + & + & 2 & 1 \\
\hline 3,8 & 1,0 & • & • & • & + & + & 2 & • & + & + & •• & • & - & • \\
\hline . & • & + & . & + & + & & & & 0,2 & 8 & 1 & 3 & + & 4 \\
\hline •. & + & 1 & - & • & + & & & & • & • & •. & + & + & + \\
\hline 2,7 & 8,7 & 5 & •. & 0,5 & 1 & & & & • & 1 & + & 1 & + & • \\
\hline 0,5 & + & + & & & & • & + & • & - & • & - & + & + & -. \\
\hline$\dot{1,1}$ & $\dot{.}$ & 1 & • & - & + & 4 & + & 3 & + & + & 2 & 1 & 9 & • \\
\hline •. & - & - & & & & & & & & & & & & \\
\hline & & & - & + & • & + & + & $\because$ & $\therefore$ & $\dot{0}$ & + & • & $\bullet$ & • \\
\hline & & & $\dot{\bullet}$ & $\dot{.}$ & + & + & $\begin{array}{l}8 \\
.\end{array}$ & 3 & 1,8 & 6 & 8 & 4 & + & + \\
\hline & & & + & •. & • & • & + & • & & & & & & \\
\hline & & & $\bullet$ & $\begin{array}{l}\bullet \\
+\end{array}$ & $\begin{array}{l}+ \\
+\end{array}$ & + & • & • & $t$ & • & • & $\bullet$ & • & $\bullet$ \\
\hline & & & + & + & 1 & & & & & & & & & \\
\hline & & & + & $\bullet$ & $\bullet$ & + & • & • & & & & & & \\
\hline & & & & & & & & & 2,7 & + & + & $t$ & $\bullet$ & $\bullet$ \\
\hline & & & & & & & & & + & 2,7 & + & + & + & + \\
\hline & & & & & & & & & + & 1,6 & 1 & 3 & + & + \\
\hline & & & & & & & & & $\bullet$ & + & + & + & • & • \\
\hline & & & & & & & & & 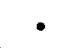 & - & - & + & . & - \\
\hline
\end{tabular}




\section{ANNEXE 2}

Tableau floristique des espèces présentes dans la séquénce de pâturage: Présence. Abondance (Contribution spécifique aut recouvrement).

Floristical table of species in the grazing sequence : Presence. Abundance (recoveral).

\begin{tabular}{|c|c|c|c|c|c|c|c|c|c|}
\hline Zone & $(0)$ & (1) & (2) & \multicolumn{2}{|c|}{ (3) } & \multicolumn{2}{|c|}{ (4) } & \multicolumn{2}{|c|}{ (5) } \\
\hline Répétition & & & & a & $b$ & a & $\mathrm{b}$ & a & $\bar{b}$ \\
\hline Litière et terre une & 9 & 14 & 26 & 14 & 38 & 19 & 27 & 11 & 16 \\
\hline $\begin{array}{l}\text { Strate arborescente } \\
\text { Pinus naritima .... }\end{array}$ & + & + & + & + & + & + & + & + & + \\
\hline $\begin{array}{l}\text { Strate arbustive } \\
\text { Ulex nanus .......... } \\
\text { Erica scoparia ......... } \\
\text { Rhamnus frangula ..... }\end{array}$ & $\begin{array}{l}+ \\
+ \\
+\end{array}$ & $\begin{array}{r}1 \\
3 \\
+\end{array}$ & $\begin{array}{l}3 \\
+ \\
+ \\
\end{array}$ & $\begin{array}{r}1 \\
2 \\
+\end{array}$ & $\begin{array}{l}3 \\
3 \\
1 \\
\end{array}$ & $\begin{array}{r}2 \\
1 \\
+ \\
\end{array}$ & $\begin{array}{c}+ \\
1 \\
+\end{array}$ & $\begin{array}{l}+ \\
+ \\
+\end{array}$ & $\begin{array}{l}+ \\
+ \\
+\end{array}$ \\
\hline 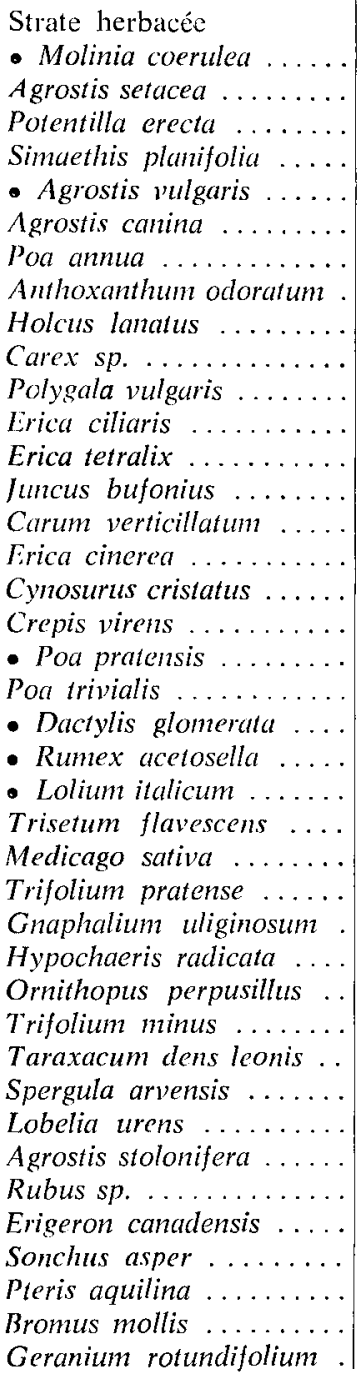 & $\begin{array}{l}88 \\
3 \\
+ \\
+\end{array}$ & $\begin{array}{c}76 \\
1 \\
+ \\
+ \\
+ \\
+ \\
3 \\
1 \\
+ \\
+ \\
1 \\
1\end{array}$ & $\begin{array}{r}60 \\
1 \\
2\end{array}$ & $\begin{array}{l}+ \\
+ \\
+ \\
+ \\
2 \\
2 \\
+ \\
+ \\
+\end{array}$ & $\begin{array}{r}1 \\
+ \\
+ \\
+ \\
1 \\
1 \\
+\end{array}$ & $\begin{array}{c}37 \\
1 \\
+ \\
+ \\
1 \\
1 \\
4 \\
+ \\
6 \\
+\end{array}$ & $\begin{array}{r}1 \\
5 \\
+ \\
5 \\
15\end{array}$ & $\begin{array}{c}10 \\
+ \\
3 \\
+ \\
59 \\
2\end{array}$ & $\begin{array}{r}+ \\
+ \\
3 \\
3 \\
34 \\
3\end{array}$ \\
\hline
\end{tabular}

Zone (0): Jamais pâturée ;

(1) : Hors pâturage et hivernage depuis avril 1977 ;

(2) : Hors pâturage et hivernage depuis mai 1978;

(3) : De pâturage estival et d'hivernage en forte charge ;

(4) : Périphérique à la zone de ravitaillement;

(5) : De ravitaillement. 